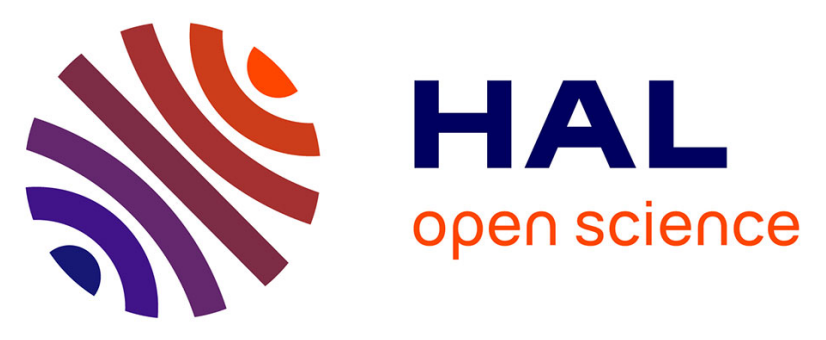

\title{
Human and mouse granzyme $M$ display divergent and species-specific substrate specificities
}

Stefanie Adriana Hendrika de Poot, Marijn Westgeest, Daniel R. Hostetter, Petra van Damme, Kim Plasman, Kimberly Demeyer, Roel Broekhuizen, Kris Gevaert, Charles S. Craik, Niels Bovenschen

\section{To cite this version:}

Stefanie Adriana Hendrika de Poot, Marijn Westgeest, Daniel R. Hostetter, Petra van Damme, Kim Plasman, et al.. Human and mouse granzyme M display divergent and species-specific substrate specificities. Biochemical Journal, 2011, 437 (3), pp.431-442. 10.1042/BJ20110210 . hal-00608385

\section{HAL Id: hal-00608385 https://hal.science/hal-00608385}

Submitted on 13 Jul 2011

HAL is a multi-disciplinary open access archive for the deposit and dissemination of scientific research documents, whether they are published or not. The documents may come from teaching and research institutions in France or abroad, or from public or private research centers.
L'archive ouverte pluridisciplinaire HAL, est destinée au dépôt et à la diffusion de documents scientifiques de niveau recherche, publiés ou non, émanant des établissements d'enseignement et de recherche français ou étrangers, des laboratoires publics ou privés. 


\title{
Human and mouse granzyme $M$ display divergent and species-specific
}

\section{substrate specificities}

Stefanie A.H. de Poot $^{1^{*}}$, Marijn Westgeest ${ }^{1^{*}}$, Daniel R. Hostetter ${ }^{2}$, Petra Van Damme ${ }^{3,4}$, Kim Plasman $^{3,4}$, Kimberly Demeyer ${ }^{3,4}$, Roel Broekhuizen ${ }^{1}$, Kris Gevaert ${ }^{3,4}$, Charles S. Craik ${ }^{2}$, Niels Bovenschen $^{1}$

1 Department of Pathology, University Medical Center Utrecht, Utrecht, The Netherlands

2 Department of Pharmaceutical Chemistry, University of California, San Francisco, USA

3 Department of Medical Protein Research, VIB, B-9000 Ghent, Belgium

4 Department of Biochemistry, Ghent University, B-9000, Ghent, Belgium

Address correspondence to: Dr. N. Bovenschen, Department of Pathology, University Medical Center Utrecht, Heidelberglaan 100, 3584 CX Utrecht, The Netherlands, Telephone: +31-88-

7553889, Fax: +31-30-2544990, E-mail: N.Bovenschen@umcutrecht.nl

*These authors contributed equally to this work

Running title: Functional divergence of human and mouse GrM

\begin{abstract}
Abbreviations: COFRADIC, combined fractional diagonal chromatography; GrA, granzyme A; $\mathrm{GrB}$, granzyme B; GrM, granzyme M; GrM-SA, GrM with $\mathrm{S}^{195} \mathrm{~A}$ mutation in catalytic center; hGrM, human GrM; NK cell, natural killer cell; NPM, nucleophosmin; mGrM, mouse GrM; PI, propidium iodide; pNA, $p$-nitroanalide; SILAC, stable isotope labeling by amino acids in cell culture; SLO, streptolysin O; 2D-DIGE, two-dimensional difference gel electrophoresis.
\end{abstract}




\begin{abstract}
Cytotoxic lymphocyte protease granzyme $\mathrm{M}(\mathrm{GrM})$ is a potent inducer of tumor cell death and a key regulator of inflammation. Although human GrM (hGrM) and mouse GrM (mGrM) display extensive sequence homology, the substrate specificity of mGrM remains unknown. Here, we show that hGrM and mGrM have diverged during evolution. Positional scanning libraries of tetrapeptide substrates revealed that mGrM preferred to cleave after a Met residue, whereas hGrM clearly favors a Leu at the P1 position. The kinetic optimal nonprime subsites of both granzymes were also distinct. Gel-based and complementary positional proteomics showed that hGrM and mGrM have a partially overlapping set of natural substrates and a diverged prime and nonprime consensus cleavage motif with Leu and Met being major P1 determinants. Consistent with positional scanning libraries of tetrapeptide substrates, P1 Met was more frequently used by mGrM as compared with hGrM. Both hGrM and mGrM cleaved $\alpha$-tubulin with similar kinetics. Strikingly, neither hGrM nor mGrM hydrolyzed mouse nucleophosmin, whereas human nucleophosmin was hydrolyzed efficiently by GrM from both species. Replacement of the putative $\mathrm{P} 1$ '-P2' residues in mouse nucleophosmin with the corresponding residues of human nucleophosmin restored cleavage of mouse nucleophosmin by both granzymes. This further demonstrates the importance of prime sites as structural determinants for GrM substrate specificity. GrM from both species efficiently triggered apoptosis in human but not in mouse tumor cells. These data indicate that hGrM and mGrM not only exhibit divergent specificities, but also trigger species-specific functions.
\end{abstract}

Keywords: granzyme M, $\alpha$-tubulin, nucleophosmin, apoptosis, proteomics, protease, substrate specificity, degradomics, $\mathrm{N}$ - and $\mathrm{C}$-terminal COFRADIC.

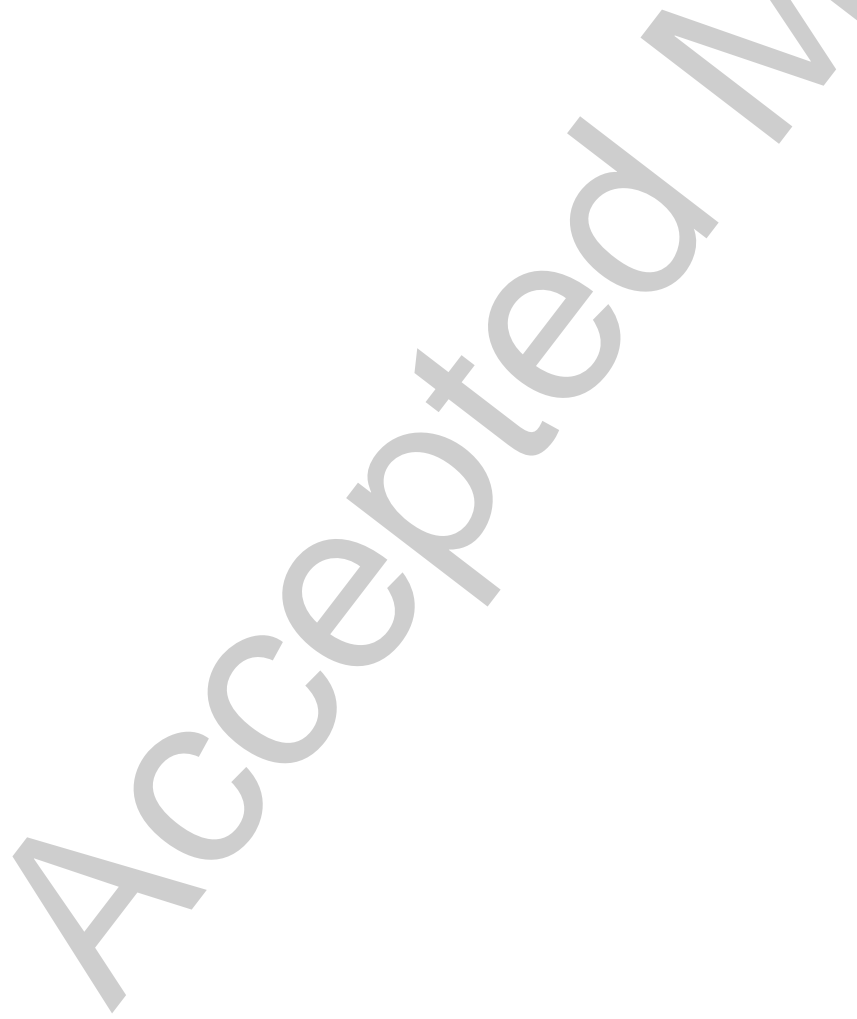




\section{Introduction}

The first line of defense against tumor and virus-infected cells is formed by cytotoxic $\mathrm{T}$ lymphocytes and natural killer (NK) cells $[1,2]$. Upon recognition of a target cell, these cytotoxic lymphocytes can initiate target cell death via either the death receptor [3] or the granule exocytosis pathway [4]. In the granule-exocytosis pathway, cytotoxic lymphocytes secrete a family of granule-associated serine proteases known as granzymes, and the pore-forming protein perforin [5]. Perforin facilitates the entry of granzymes into the target cell, enabling granzymes to induce cell death by cleaving intracellular substrates. In humans, five granzymes have been identified (GrA, GrB, GrH, GrK, and GrM) [6-8]. The mechanisms via which GrA and GrB induce cytotoxicity have been studied extensively, whereas far less is known about the other human granzymes [9]. Recently, granzymes have also been postulated to play key roles in regulating inflammation $[10,11]$.

Human granzyme $\mathrm{M}$ (hGrM) [12] is unique in that it preferably cleaves after a Leu residue $[13,14]$ and is highly expressed in NK cells and to a lesser extent in CD8+ T cells $[15,16]$. hGrM is a potent and efficient inducer of tumor cell death in vitro and in vivo [17, 18]. This cell death is characterized by rapid cell swelling, formation of large cytoplasmic vacuoles, chromatin condensation with only slight segmentation of the nuclei, and finally lysis of the cells [17]. The molecular mechanisms by which hGrM initiates cell death remain controversial in literature. hGrM has been demonstrated to trigger cell death in a caspase-independent fashion, with no fragmentation of DNA, no formation of reactive oxygen species (ROS), and no perturbation of mitochondria $[17,19,20]$. In these studies, hGrM has been demonstrated to cleave the microtubule network component $\alpha$-tubulin, leading to disorganization of the microtubule network [19], and nucleophosmin/B23 (NPM), a multifunctional phosphoprotein essential for cell survival [20]. In contrast, Fan and colleagues have reported that hGrM promotes cell death in a manner similar to GrB, including caspase-3 activation, DNA fragmentation, generation of ROS, and cytochrome $c$ release from the mitochondria, through cleavage of heat shock protein (HSP)-75, ICAD, poly(ADP-ribose) polymerase (PARP), and survivin [21-23].

While there are five granzymes in humans, mice express at least ten granzymes $(\mathrm{A}, \mathrm{B}, \mathrm{C}, \mathrm{D}$, $\mathrm{E}, \mathrm{F}, \mathrm{G}, \mathrm{K}, \mathrm{M}$ and N) [7]. hGrM has an ortholog in mice and mouse GrM (mGrM) functions in vivo have been studied in a mGrM knockout mouse model $[10,18,24]$. Like hGrM, mGrM has been implicated to participate in tumor clearance [18]. More recently mGrM has also been proposed to play a key role in the regulation of inflammation in vivo via yet to be established mechanisms [10]. Although hGrM and mGrM display extensive sequence homology, very little is known about the specificity of the mGrM protease. In the present study, we show that hGrM and mGrM exhibit divergent and species-specific substrate specificities. hGrM and mGrM display distinct P1 and subsite preferences, have narrow macromolecular substrate specificities that overlap only partially, cleave human but not mouse NPM, and trigger apoptosis in certain human but not mouse tumor cell lines. This stresses that caution is needed when using mouse models to elucidate GrM functions in humans.

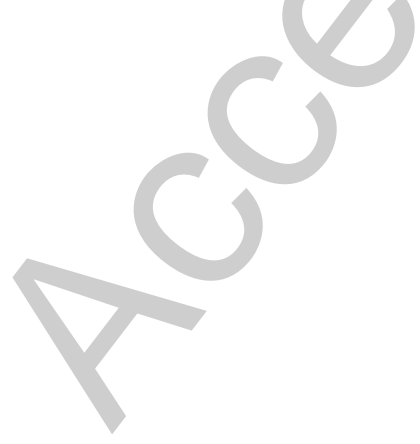




\section{Experimental Procedures}

Cell lines, Antibodies, and Reagents - Human HeLa and murine $\mathrm{C}_{2} \mathrm{C}_{12}$ cells were grown in DMEM medium, supplemented with $10 \%$ fetal calf serum, 100 units $/ \mathrm{mL}$ penicillin, and $100 \mu \mathrm{g} / \mathrm{mL}$ streptomycin (Invitrogen). For the $\mathrm{N}$ - and C-terminal combined fractional diagonal chromatography (COFRADIC) analyses, human K-562 cells were grown in RPMI 1640 glutamax medium, supplemented with 10\% dialyzed fetal bovine serum, 100 units/mL penicillin, and 100 $\mu \mathrm{g} / \mathrm{ml}$ streptomycin containing either $57.5 \mu \mathrm{M}$ natural, ${ }^{13} \mathrm{C}_{6}$ or ${ }^{13} \mathrm{C}_{6}{ }^{15} \mathrm{~N}_{4}$ L-arginine (Cambridge Isotope Labs). Cells were passaged for at least six population doublings for complete incorporation of the labeled arginine. Cell-free protein extracts were generated from exponentially growing HeLa, $\mathrm{C}_{2} \mathrm{C}_{12}$ and K-562 cells. Cells $\left(10^{8}\right.$ cells $\left./ \mathrm{ml}\right)$ were washed two times in a buffer containing either $50 \mathrm{mM}$ Tris (pH 7.4) and $150 \mathrm{mM} \mathrm{NaCl}\left(\mathrm{HeLa}\right.$ and $\mathrm{C}_{2} \mathrm{C}_{12}$ cells) or 50 $\mathrm{mM}$ Tris ( $\mathrm{pH} 8.0)$ and $100 \mathrm{mM} \mathrm{NaCl}$ (K-562 cells), and lysed in the same buffer by three cycles of freeze-thawing. Samples were centrifuged for $10 \mathrm{~min}$ at $20,000 \mathrm{~g}$ at $4{ }^{\circ} \mathrm{C}$ and cell-free protein extracts were stored at $-80^{\circ} \mathrm{C}$. Protein concentration was quantified by the Bradford method. Antibodies used were anti- $\alpha$-tubulin clone B-5-1-2 (Sigma), anti- $\beta$-tubulin clone TUB 2.1 (Sigma), and anti-nucleophosmin clone FC-61991 (Invitrogen). SLO was purchased from Aalto Bio Reagents. Homology modeling was performed using SWISS-MODEL [25].

Recombinant proteins - The cDNA encoding mature mGrM (residues $\mathrm{Ile}^{27}-\mathrm{Val}^{264}$ ) was amplified from mouse thymus cDNA (MD-702, Zyagen) using the oligonucleotides 5'CCGCTCGAGAAACGTATCATTGGGGGTCGAG-3' 5 and 'TAAAGCGGCCGCCTTAGACCAAAGATTGGGG-3', and cloned into yeast expression vector pPIC9 (Invitrogen). Catalytically inactive mouse GrM-SA, in which the $\mathrm{Ser}^{195}$ residue in the catalytic center is replaced by Ala, was generated by site-directed mutagenesis (Stratagene). Plasmids were transformed into the GS115 (his4) strain of Pichia pastoris. hGrB, hGrM and mGrM and the catalytically inactive GrM-SA mutants were produced in Pichia pastoris and purified using cation-exchange chromatography as described previously [19]. GrM fractions were dialyzed against $50 \mathrm{mM}$ Tris ( $\mathrm{pH} 7.4)$, and $150 \mathrm{mM} \mathrm{NaCl}$ and stored at $-80^{\circ} \mathrm{C}$. GrM activity was determined using the synthetic chromogenic substrates Suc-Ala-Ala-Pro-Leu- $p$-nitroanalide (Bachem) and Suc-Lys-Val-Pro-Leu- $p$-nitroanalide (GL Biochem). To directly compare hGrM and $\mathrm{mGrM}$ substrate specificity, both enzymes were titrated using the synthetic chromogenic substrate Suc-Ala-Ala-Pro-Leu- $p$-nitroanalide. hGrM was twice as efficient as its mouse counterpart in cleaving this substrate. The catalytically inactive GrM-SA mutants of both hGrM and mGrM did not show any activity (data not shown). Human and mouse granzyme concentrations in all experiments were matched based on Suc-Ala-Ala-Pro-Leu-pNA hydrolysis. Human $\alpha$-tubulin, mouse $\alpha$-tubulin, human NPM and mouse NPM cDNA's were amplified from IMAGE clones 3871729, 6306481, 5575414 and 30438901, respectively, and cloned into the bacterial expression vector pQE80L. N-terminally His-tagged $\alpha$-tubulin and His-tagged NPM were expressed in Escherichia coli strain BL21 as recommended by the manufacturer (Roche). The NPM P1', P2', and P1'/P2' mutants were generated by site-directed mutagenesis (Stratagene). Recombinant $\alpha$-tubulin and NPM proteins were purified by metal-chelate chromatography (Clontech), dialyzed against phosphate-buffered saline, and stored at $-80^{\circ} \mathrm{C}$.

Granzyme killing assays - HeLa and $\mathrm{C}_{2} \mathrm{C}_{12}$ cells were grown to confluence in a 96-well tissue-culture plate. Cells were washed twice in serum-free DMEM, after which they were incubated at $37^{\circ} \mathrm{C}$ with a sublytic dose of SLO (Jurkat: $0.25 \mu \mathrm{g} / \mathrm{ml} \mathrm{SLO;} \mathrm{HeLa,} \mathrm{PC3:} 0.5 \mu \mathrm{g} / \mathrm{ml}$ SLO; LR7, C26, $\mathrm{C}_{2} \mathrm{C}_{12}: 1 \mu \mathrm{g} / \mathrm{ml}$ SLO) and indicated concentrations of granzyme in a buffer containing $20 \mathrm{mM}$ Tris and $150 \mathrm{mM} \mathrm{NaCl}$ for $30 \mathrm{~min}$. The cells were washed twice with supplemented DMEM, after which the cells were incubated for another $20 \mathrm{~h}$ at $37^{\circ} \mathrm{C}$. Cell viability was assessed using flow cytometry. Cells were stained with annexinV-fluos (Invitrogen) and PI for $15 \mathrm{~min}$ in a buffer containing $140 \mathrm{mM} \mathrm{NaCl}, 4 \mathrm{mM} \mathrm{KCl}, 0.75 \mathrm{mM} \mathrm{MgCl}, 1.5 \mathrm{mM}$ $\mathrm{CaCl}_{2}$, and $10 \mathrm{mM}$ HEPES (pH 7.4). Flow cytometry was performed on a FACSCalibur (BD Biosciences) and data was analyzed using CellQuest Pro software (BD Biosciences). Cells that 
were negative for both AnnexinV and PI were regarded as living cells. The percentage of viable cells after treatment with SLO only was set at $100 \%$ and the percentages of viable cells in other conditions were calculated accordingly.

Single substrate kinetics - Granzyme activity was monitored by the synthetic chromogenic substrates Suc-Ala-Ala-Pro-Leu- $p$-nitroanalide (Bachem) and Suc-Lys-Val-Pro-Leu- $p$ nitroanalide (GL Biochem Ltd) in $100 \mathrm{mM}$ HEPES (pH 7.4), $200 \mathrm{mM} \mathrm{NaCl}$ at $37^{\circ} \mathrm{C}$. $\mathrm{GrM}$ concentration was $100 \mathrm{nM}$ and substrate concentration ranged from 0.5-3 mM. Hydrolysis of $p$ nitroanalide substrates was monitored spectrophotometrically at $405 \mathrm{~nm}$ on an Anthos Zenyth 340 rt microtiter plate reader (Anthos). Kinetic parameters $\left(\mathrm{k}_{\mathrm{cat}} / \mathrm{K}_{\mathrm{M}}\right)$ were determined using standard Michaelis-Menten kinetic equations.

Positional scanning synthetic combinatorial libraries - The preparation and characterization of the P1-diverse and P1-Met libraries of 7-amino-4-carbamoylmethylcoumarin (ACC) substrates used in this study are described elsewhere $[26,27] .10^{-10} \mathrm{~mol}$ of each well of the P1-diverse stock library was added to 20 wells of a 96-well microfluor plate. Final concentration of each substrate in the assay was $250 \mu \mathrm{M}$. Assays were initiated by addition of $5.9 \mu \mathrm{M} \mathrm{GrM}$ and were conducted at $25^{\circ} \mathrm{C}$ in buffer containing $50 \mathrm{mM}$ Tris $(\mathrm{pH} \mathrm{7.4)}$ ) and $150 \mathrm{mM} \mathrm{NaCl}$ for $2 \mathrm{~h}$. Hydrolysis of substrates was monitored fluorimetrically with an excitation wavelength of $380 \mathrm{~nm}$ and an emission wavelength of $460 \mathrm{~nm}$ on a Spectramax Gemini microtiter plate reader (Molecular Devices).

Fluorescent two-dimensional difference gel electrophoresis (2D-DIGE) - For 2D-DIGE, 100 $\mu \mathrm{g}$ of $\mathrm{HeLa}$ or $\mathrm{C}_{2} \mathrm{C}_{12}$ cell-free protein extract was incubated for $1 \mathrm{~h}$ at $37{ }^{\circ} \mathrm{C}$ with $1 \mu \mathrm{M}$ of hGrM or mGrM or their catalytically inactive mutants. Samples were precipitated, solubilized, labeled, rehydrated and isoelectrically focused as we described previously [28]. The strips were reduced and overlaid on a $12 \%$ SDS-PAGE gel. The images were acquired on a Typhoon 9410 scanner (GE Healthcare). Each condition was performed at least five times, and a dye swap was included to exclude preferentially labeled proteins from the analysis. The relative quantification of matched gel features was performed using Decyder DIA and BVA software (GE Healthcare). For inter-gel analyses, the internal standard method was used as described previously [29]. Statistical analysis was performed using the Student's t-test. $\mathrm{p}<0.05$ was regarded as statistically significant.

$\mathrm{N}$ - and C-terminal COFRADIC: isolation of terminal peptides - For N- and C-terminal COFRADIC, $1250 \mu \mathrm{g}$ of a K-562 cell-free protein extract was either left untreated $\left({ }^{13} \mathrm{C}_{6}{ }^{15} \mathrm{~N}_{4} \mathrm{~L}\right.$ arginine labeled sample), treated with $200 \mathrm{nM}$ recombinant $\mathrm{hGrM}\left({ }^{12} \mathrm{C}_{6} \mathrm{~L}\right.$-arginine labeled sample) or recombinant $\mathrm{mGrM}\left({ }^{13} \mathrm{C}_{6} \mathrm{~L}\right.$-arginine labeled sample) for $1 \mathrm{~h}$ at $37^{\circ} \mathrm{C}$. Following protease incubation, guanidinium hydrochloride was added to the cell lysates to a final concentration of $4 \mathrm{M}$ in order to denature and inactivate the proteases. The protein samples were reduced and $S$-alkylated, followed by tri-deuteroacetylation of primary amines and trypsin digestion as described previously [30, 31]. $\mathrm{N}$ - and $\mathrm{C}$ - terminal COFRADIC analyses were performed as described in [32]. In this setup, ${ }^{12} \mathrm{C}_{4}$-butyrylation was used for the hGrM-treated sample, ${ }^{13} \mathrm{C}_{4}$-butyrylation for the mGrM-treated sample and ${ }^{13} \mathrm{C}_{2}$-butyrylation for the control sample.

LC-MS/MS analysis - LC-MS/MS analysis was performed using an Ultimate 3000 HPLC system (Dionex, Amsterdam, The Netherlands) in-line connected to a LTQ Orbitrap XL mass spectrometer (Thermo Electron, Bremen, Germany) and, per LC-MS/MS analysis, $5 \mu 1$ of sample ( $1 / 4^{\text {th }}$ of total sample) was consumed. LC-MS/MS analysis and generation of MS/MS peak lists were performed as described in [33]. These MS/MS peak lists were then searched with Mascot using the Mascot Daemon interface (version 2.2.0, Matrix Science). Searches were performed in the Swiss-Prot database with taxonomy set to human (UniProtKB/Swiss-Prot database version 2010_10 containing 20,258 human protein sequences). Trideutero-acetylation at lysines, carbamidomethylation of cysteine, and methionine oxidation to methionine-sulfoxide were set as fixed modifications. Variable modifications were trideutero-acetylation and acetylation of protein 
N-termini. Semi-ArgC was set as the used protease (no missed cleavages were allowed) and the mass tolerance on the precursor ion was set to $10 \mathrm{ppm}$ and on fragment ions to $0.5 \mathrm{Da}$. In addition, Mascot's C13 setting was set to 1. Only MS/MS spectra that exceeded the corresponding Mascot threshold score of identity (at 95\% confidence level) were withheld. The estimated false discovery rate by searching decoy databases was typically found to lie between 2 and $4 \%$ on the spectrum level [30]. All quantifications [SILAC $\left({ }^{12} \mathrm{C}_{6} \mathrm{~L}\right.$-arginine versus ${ }^{13} \mathrm{C}_{6} \mathrm{~L}$ arginine, ${ }^{12} \mathrm{C}_{6} \mathrm{~L}$-arginine versus ${ }^{13} \mathrm{C}_{6}{ }^{15} \mathrm{~N}_{4} \mathrm{~L}$-arginine and ${ }^{13} \mathrm{C}_{6} \mathrm{~L}$-arginine versus ${ }^{13} \mathrm{C}_{6}{ }^{15} \mathrm{~N}_{4} \mathrm{~L}$ arginine ) and butyrylation $\left({ }^{12} \mathrm{C}_{4}\right.$-butyrylated versus ${ }^{13} \mathrm{C}_{4}$-butyrylated, ${ }^{12} \mathrm{C}_{4}$-butyrylated versus ${ }^{13} \mathrm{C}_{2}$-butyrylated and ${ }^{13} \mathrm{C}_{2}$-butyrylated versus ${ }^{13} \mathrm{C}_{4}$-butyrylated)] were carried out using the Mascot Distiller Quantitation Tool (version 2.2.1). The quantification method details were as follows: constrain search: yes; protein ratio type: average; report detail: yes; minimum peptides: 1; protocol: precursor; allow mass time match: yes; allow elution shift: no; all charge states: yes; fixed modifications: mass values. Ratios for the proteins were calculated by comparing the XIC peak areas of all matched light versus medium, light versus heavy and medium versus heavy peptides. The calculated ratios that were reported as FALSE were all verified by visual inspection of all highest scoring MS spectra. 


\section{Results}

The primary and extended specificities of human and mouse GrM are distinct - hGrM and its mouse ortholog display a considerable sequence homology of 69\% (Fig. 1A). Whereas the Ser, His, and Asp residues of the catalytic triad are completely conserved between hGrM and $\mathrm{mGrM}$, several residues that are predicted to play key roles in the substrate recognition of hGrM (34) differ between both granzymes (Fig. 1A). These differences result in an altered substrate binding pocket in mGrM as can be visualized in a homology model of mGrM based on the known crystal structure of hGrM (Fig. 1B) [34]. hGrM prefers to cleave after a Leu residue at the Pl position (nomenclature for amino acid positions in substrates is $\mathrm{P} n-\mathrm{P} 2-\mathrm{P} 1-\mathrm{P} 1$ ' $-\mathrm{P} 2$ '- $-\mathrm{P} n$ ', with amide bond hydrolysis occurring after P1, and the corresponding enzyme binding sites denoted as Sn-S2-S1S1'-S2'-Sn') [35]. The optimal P4-P1 tetrapeptide specificity of hGrM has previously been identified as Lys-Val-Pro-Leu $[13,14]$. To test whether the tetrapeptide specificity of mGrM is similar to that of hGrM, mGrM was incubated with the chromogenic optimal human tetrapeptide substrate Suc-KVPL-pNA (Fig. 1C). While hGrM efficiently hydrolyzed this substrate $\left(\mathrm{k}_{\text {cat }} / \mathrm{K}_{\mathrm{M}}=\right.$ $\left.6.6 \times 10^{3} \mathrm{M}^{-1} \mathrm{~s}^{-1}\right)$, mGrM was approximately 4.5 -fold less efficient $\left(\mathrm{k}_{\mathrm{cat}} / \mathrm{K}_{\mathrm{M}}=1.5 \times 10^{3} \mathrm{M}^{-1} \mathrm{~s}^{-1}\right)$. This suggests that hGrM and mGrM are indeed structurally distinct proteases with distinct substrate binding pockets. The primary specificity of $\mathrm{mGrM}$ was profiled using a positional scanning synthetic combinatorial library (PS-SCL) of tetrapeptide coumarin substrates known as the P1-diverse library $[14,27]$. For hGrM, this method has previously demonstrated a strong P1 preference for Leu $(100 \%)$ over Met $(\sim 16 \%)$ and non-physiological Nle $(\sim 46 \%)$ and no tolerance for other residues at this position $[13,14]$. In contrast to $\mathrm{hGrM}, \mathrm{mGrM}$ displayed a preference for Nle $(100 \%)$ and Met ( $\sim 95 \%)$ over Leu ( 68\%) (Fig. 1D). The residues Ala, Gln, His, Lys, Phe, Ser, Trp and Tyr were also tolerated at the P1 position, although to a lesser extent. The P4-P2 extended substrate specificity of mGrM was determined using a P1-fixed Met PS-SCL library (Fig. 1D). Strikingly, whereas hGrM shows an almost absolute requirement for Pro at the P2 with some tolerance for Ala [13], mGrM clearly preferred Ala over Pro and displayed a broad tolerance for other amino acids at P2. While the preferred P3 residues of $\mathrm{mGrM}$ differed slightly from those of hGrM, both granzymes seemed to favor Glu or Val at P3. At the P4 position, mGrM was highly tolerant to a large number of amino acids, while hGrM shows a distinct preference for either a Lys, Nle or a His at the P4 [13]. Together, these data indicate that hGrM and $\mathrm{mGrM}$ differ in their primary and P4-P2 substrate specificity.

Human and mouse GrM display restricted macromolecular substrate specificities that overlap only partially - To compare the macromolecular substrate specificities of hGrM and $\mathrm{mGrM}$, two different proteomics-based strategies were applied. First, a fluorescence twodimensional difference gel electrophoresis (2D-DIGE) proteomic approach was employed that scans the native proteome of tumor cells for macromolecular substrates of both granzymes and directly determines the efficiency of substrate cleavage (Fig. 2). Cell lysates of human cervix carcinoma (HeLa) and mouse myoblast $\left(\mathrm{C}_{2} \mathrm{C}_{12}\right)$ cells were incubated with hGrM, hGrM-SA, $\mathrm{mGrM}$ or mGrM-SA and were subsequently labeled with either a red fluorescent dye (Cy5) or a green fluorescent dye (Cy3). Two representative 2D-DIGE gels of cell lysates after hGrM (Fig. 2A) or mGrM (Fig. 2B) treatment are shown. GrM-treated lysates in these gels were labeled green, while the lysates that had been treated with GrM-SA were labeled red. Spots present in greater abundance in the control sample appear red and indicate possible intact GrM substrates, whereas spots present in greater abundance in the granzyme-treated sample appear green and reflect the appearance of specific cleavage products (the unaffected proteome appears yellow). Approximately 3,000 protein spots were resolved from the human HeLa cell lysate, of which 44 spots clearly appeared (cleavage products) and $40(\sim 1.3 \%)$ decreased in intensity with different efficiencies (intact substrates) after hGrM treatment (Fig. 2A) (log peak volume change $>2$-fold, $\mathrm{p}<0.05$ ). For mouse $\mathrm{C}_{2} \mathrm{C}_{12}$ cell lysates, about 2,300 proteins spots were resolved. After mGrM treatment, 39 protein spots clearly appeared (cleavage products) and $12(\sim 0.5 \%)$ decreased in intensity with different efficiencies (intact substrates) (Fig. 2B) (log peak volume change $>2$-fold, 
$\mathrm{p}<0.05$ ). This relatively low number of recognized substrates for hGrM and mGrM suggests that the macromolecular substrate specificities of both granzymes are highly restricted. Interestingly, 17 out of the 40 macromolecular substrates that could be detected were cleaved and shared by both granzymes in HeLa lysates (log peak volume change $>2$-fold, $p<0.05$ ), indicating a $-42 \%$ overlap in macromolecular substrate specificity (Fig. 2C). For the cleavage fragments, 36 out of 51 spots with at least a 2 -fold log peak volume change were shared, strongly suggesting that part of the detected cleavage events occurred at the same P1 cleavage sites, while for the selection criteria applied, other events were considered unique for mGrM or hGrM. In the $\mathrm{C}_{2} \mathrm{C}_{12}$ lysate, 11 out of 32 proteins were cleaved by both granzymes, indicative of a $\sim 34 \%$ overlap in macromolecular substrate specificity, while 19 out of 61 cleavage fragments overlapped $(\sim 31 \%)$ (Fig. 2D).

To further probe the macromolecular differences in substrate selection among the human and mouse granzyme $\mathrm{M}$ orthologs, we made use of the COFRADIC-based complementary positional proteomics approach to study GrM-specific proteolysis in human K-562 cell lysates. This approach allows us to identify and to compare the (consensus) cleavage sites of hGrM and $\mathrm{mGrM}$, but does not directly determine efficiencies of cleavage events. In analogy with previous GrB setups analyzed [32, 36], a SILAC [37] ${ }^{12} \mathrm{C}_{6}$-L-Arg and ${ }^{13} \mathrm{C}_{6}$-L-Arg labeled cell lysate served as a hGrM and mGrM substrate pool respectively, while a ${ }^{13} \mathrm{C}_{6}{ }^{15} \mathrm{~N}_{4}-\mathrm{L}-\mathrm{Arg}$ labeled proteome served as control. This allowed quantification of (neo-)N-terminal peptides since all samples were subject to tryptic digestion and therefore end on arginine. We further differentially tagged protein C-terminal peptides using NHS-esters of ${ }^{12} \mathrm{C}_{4}(\mathrm{hGrM}),{ }^{13} \mathrm{C}_{2}$ (control) or ${ }^{13} \mathrm{C}_{4}$ butyric acid (mGrM). Following LC-MS/MS analysis, we here identified 720 unique hGrM and/or mGrM specific cleavage sites in 488 protein substrates based on their corresponding 577 neo-N and/or 155 neoC-terminal peptide(s) (Supplemental Table 1). Of these, $411 \mathrm{hGrM}$ and/or $\mathrm{mGrM}$ specific cleavage sites were generated by processing at Leu $(57 \%), 75$ by cleavage after Met $(10 \%)$ and the remaining 234 cleavage sites $(33 \%)$ were, consistent with the positional scanning results, raised upon processing at alternative P1-specificities, including Ala, Cys and Gln. Besides monitoring of (neo-)termini generated by GrM, the differential labeling strategies applied allow for a direct comparison of differences in substrate specificity profiles for both granzymes. As such, single ${ }^{12} \mathrm{C}_{6} \mathrm{~L}$-Arg neo-N- or ${ }^{12} \mathrm{C}_{4}$-butyrylayed neo-C-termini, and ${ }^{13} \mathrm{C}_{6} \mathrm{~L}$-Arg neo-N- or ${ }^{13} \mathrm{C}_{4-}$ butyrylayed neo-C-termini indicate respectively unique hGrM and $\mathrm{mGrM}$ substrates, while couples spaced by six or four mass units respectively indicate neo-N- or neo-C-termini raised by both orthologs (Figure 3). The ratio of ion signal intensities of such couples is further indicative of the difference in substrate cleavage efficiency between hGrM and mGrM. Of all sites identified, 481 sites $(67 \%)$ in 359 substrates were found to be cleaved by both proteases, while 230 (196 proteins) and 9 cleavages ( 9 proteins) were uniquely introduced by the action of hGrM and mGrM respectively (Supplemental Table 1). As deduced from the neo-termini specific $\mathrm{hGrM} / \mathrm{mGrM}$ ratios, on average, $\mathrm{hGrM}$ seemed to be 4 to 5 fold more efficient in cleaving its substrates as compared to mGrM.

The COFRADIC-based complementary positional proteomics approach allows us to directly compare consensus cleavage sites of hGrM and mGrM. The general amino acid conservation in the set of GrM substrates with a Leu or Met at P1 that were more efficiently cleaved by hGrM than by mGrM is shown in Fig. 4A. The general amino acid conservation in the set of more efficiently cleaved mGrM substrates with P1 Leu or Met is shown in Fig. 4B. Overall, the identified P1-P4 specificity of mGrM is consistent with our PS-SCL data (Fig. 1D). To further distinguish between differentially accommodated amino acids from $\mathrm{P} 4$ to $\mathrm{P} 4$ ' in their respective subsites of hGrM and mGrM, a differential iceLogo [38] was created using the human and mouse Leu and Met P1-specific data subsets for which the hGrM or mGrM cleavage efficiency (as deduced from the proteomics data) was at least $50 \%$ more efficient as compared to the mean hGrM versus $m G r M$ cleavage ratio observed (Fig. 4C). Statistically significant residues $(p \leq 0.01)$ are plotted with the size of the amino acid proportional to the difference observed in cleavage 
efficiency between hGrM and mGrM on their respective substrates (Fig. 4C). In line with the PSSCL data, a P1 Met residue seems to be better accommodated by mGrM as compared to hGrM. Other differentially accommodated residues include Gly or Glu at P2, a P2 Gly being generally inhibitory for hGrM-specific cleavage (the increased prevalence of Gly at P2 is also apparent when comparing the P2 hGrM and mGrM PS-SCL preferences), and a Glu being inhibitory for mGrM-specific cleavage (see also representative examples Fig. 3A-F). In general, the presence of a positively charged $\mathrm{P} 4$ Lys or His is more stimulatory for hGrM-specific cleavage as compared to mGrM-specific cleavage (Arg at P4). Beyond the P4-P1 motif, the amino acid occupancy of P2' is also clearly discriminative with Leu, Phe, Tyr, and Asp being better accommodated by the mGrM S2' pocket, while Ala, Asn, Lys, Ser, Gly, and Val represent better accommodated hGrM P2' residues. In addition, Suppl. Fig. 1 plots the different amino acid occurrences in percentages at the most discriminative $\mathrm{P}$ and $\mathrm{P}^{\prime}$-positions in more efficient hGrM versus more efficient $\mathrm{mGrM}$ cleavage site motifs as bar charts.

Collectively, these data indicate that hGrM and mGrM display narrow macromolecular substrate specificities that overlap only partially. Interestingly, the hGrM and mGrM substrates that were identified by this complementary positional proteomics approach include the already known hGrM substrates $\alpha$-tubulin [19] and NPM [20] (Supplemental Table 1).

Human and mouse GrM cleave human and mouse $\alpha$-tubulin with similar efficiency - We have previously demonstrated that hGrM cleaves the microtubule component $\alpha$-tubulin, leading to a disorganization of the microtubule network that may contribute to cell death [19]. The proteolysis of $\alpha$-tubulin was further biochemically analyzed in human (HeLa) and mouse $\left(\mathrm{C}_{2} \mathrm{C}_{12}\right)$ cell lysates. Mouse $\alpha$-tubulin was cleaved by $\mathrm{mGrM}$ in a time- and concentration-dependent manner similar to the cleavage of human $\alpha$-tubulin by hGrM (Fig. 5A). Semi-quantitative analysis of the protein bands indeed showed similar kinetics of $\alpha$-tubulin cleavage by both hGrM and mGrM (Fig. 5B). $\mathrm{mGrM}$ also cleaved human $\alpha$-tubulin and vice versa with similar efficiency (Fig. 5C). To exclude the possibility that GrM-induced cleavage of $\alpha$-tubulin is indirect and to visualize GrM-induced cleavage products, these experiments were repeated using purified recombinant human and mouse His-tagged $\alpha$-tubulin. Consistent with Fig. 5A-C, both human and mouse His-tagged $\alpha$ tubulin were cleaved by both hGrM and mGrM (Fig. 5D). Despite the fact that $\alpha$-tubulin is extremely well conserved between human and mouse (99.6\% sequence homology) (Fig. 5E), the cleavage fragments that appeared after GrM treatment of purified His-tagged $\alpha$-tubulin differed between hGrM and mGrM (Fig. 5D). These data indicate that although both hGrM and mGrM cleave $\alpha$-tubulin with similar efficiency, distinct cleavage sites are preferred (See also Suppl. Table 1).

Species-specific substrate proteolysis: human and mouse GrM cleave human but not mouse nucleophosmin - The nucleolar phosphoprotein NPM is a multifunctional tumor-suppressor protein that has been directly implicated in cancer pathogenesis [39] and apoptosis [20]. Cleavage of NPM by GrM has been proposed to contribute to the mechanism by which GrM triggers tumor cell death [20]. The proteolysis of NPM by hGrM and mGrM was tested in HeLa and $\mathrm{C}_{2} \mathrm{C}_{12}$ cell lysates (Fig. 6A). As expected [20], cleavage of human NPM by hGrM was highly efficient and time- and concentration-dependent, with proteolysis almost going to completion at 15 minutes after the addition of $400 \mathrm{nM} \mathrm{hGrM}$, or at 1 hour after the addition of $50 \mathrm{nM}$ hGrM (Fig. 6B). Cleavage of mNPM, however, was far less efficient, with virtually no reduction of the full-length protein band after 2 hours incubation with $400 \mathrm{nM}$ mGrM. Kinetic analysis revealed that $\mathrm{mGrM}$ was $\sim 40$-fold less efficient in cleaving its species-matched NPM as compared with hGrM (Fig. 6B). To determine whether this marked variation in NPM cleavage was due to differences between hGrM and mGrM or due to differences between human and mouse NPM, NPM from both human and mouse cell lysates was exposed to both granzymes (Fig. 6C). Interestingly, while human NPM was hydrolyzed with similar efficiency by both hGrM and mGrM, mouse NPM was a poor substrate of both $\mathrm{mGrM}$ and hGrM. Similar results were obtained when the granzymes were incubated with purified recombinant human and mouse His-tagged NPM (Fig. 6D), 
suggesting that differences in NPM between species rather than differences in GrM explain these findings. Human and mouse NPM share a sequence similarity of $93.9 \%$ and the known hGrM cleavage site [20] (at least P9-P1) in NPM is conserved between human and mouse. Cleavage of NPM at this site was also detected in a human K-562 lysate using complementary positional proteomics (observed hGrM/mGrM ratio of 1.6, see Supplemental Table 1A-B). Notably, there are two Ala residues at the $\mathrm{P} 1$ ' and $\mathrm{P} 2$ ' positions of human NPM that are not present in mouse NPM. Instead, mouse NPM harbors the non-preferred (Fig. 4A) negatively charged Asp ${ }^{159}$ and $\mathrm{Glu}^{160}$ residues at these positions (Fig. 6E), respectively. To determine whether this difference is responsible for the impaired cleavage of mouse NPM by both mGrM and hGrM, the Asp ${ }^{159}$ and Glu $^{160}$ residues of mouse NPM were 'humanized' using site-directed mutagenesis (Fig. 6F). The efficiency of both hGrM and mGrM-mediated cleavage of mouse NPM slightly increased when the P1' residue of mouse NPM was mutated into the corresponding residue of human NPM, and the efficiency was completely restored when only the $\mathrm{P} 2$ ' residue was replaced with an Ala or when both residues were replaced simultaneously (Fig. 6G). These data show that mouse NPM cannot be cleaved by both hGrM and mGrM because of species-specific differences in the prime site residues $\mathrm{P} 1$ ' and predominantly $\mathrm{P} 2$ '. Consistent with our positional proteomics analysis, this further stresses that prime sites in macromolecular substrates are important structural determinants for GrM substrate specificity.

GrM-induced cell death is species-dependent - The functional and structural speciesdependent differences here identified between hGrM and $\mathrm{mGrM}$ prompted us to investigate the tumor cell death potential of both granzymes. Human (HeLa) and mouse $\left(\mathrm{C}_{2} \mathrm{C}_{12}\right)$ cells were incubated with purified GrM in the presence or absence of the pore-forming protein streptolysin $\mathrm{O}$ (SLO), a perforin-analogue. The cells were subsequently stained with apoptosis markers annexin $\mathrm{V}$ and propidium iodide (PI), and cell viability was assessed by means of flow cytometry. Both hGrM and mGrM efficiently induced cell death in human HeLa cells, with mGrM being slightly more potent than hGrM (Fig. 7A). Additionally, hGrM and mGrM displayed clear cytotoxic potential towards the human prostate cancer PC3 and leukaemic Jurkat cell lines (Supplemental Fig. 2). Under the same conditions, however, neither hGrM nor mGrM was able to induce apoptosis in mouse $\mathrm{C}_{2} \mathrm{C}_{12}$ cells (Fig. 7B). GrM from both species also failed to trigger apoptosis in several other mouse cell lines, including the colon carcinoma $\mathrm{C} 26$ cell line and the fibroblast LR7 cell line (Supplemental Fig. 2). The catalytically inactive GrM-SA mutants and SLO alone displayed no cytotoxicity in these experiments, while human GrB efficiently induced apoptosis in all above mentioned cell lines (Fig. 7 and Supplemental Fig. 2). These data indicate that, at least in the cell lines tested, both mGrM and hGrM have cytotoxic potential in human cells, but not in mouse cells.

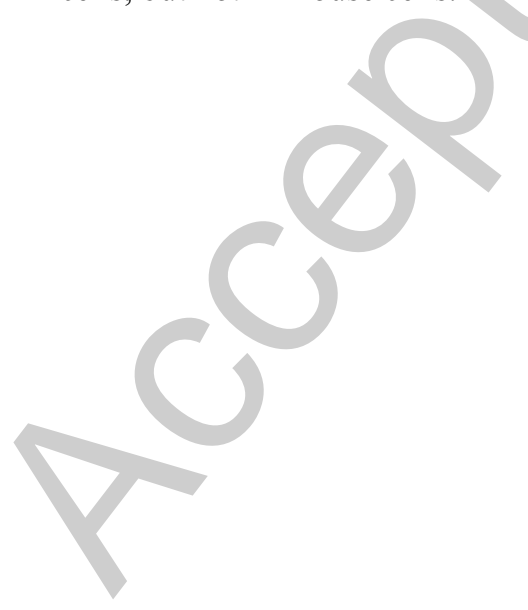




\section{Discussion}

Granzymes are key players in the effector arm of the immune response against tumor and virusinfected cells [1, 4]. Recently, it has been demonstrated that human and mouse GrB are structurally and functionally different in that $\mathrm{GrB}$ from both species cleaves pro-caspase-3, while only human GrB is able to cleave BID [36, 40-42]. In the present study, we have employed three distinct (proteomic) approaches to show that hGrM and mGrM also exhibit divergent and speciesspecific substrate specificities. mGrM and hGrM displayed distinct, but to some extent overlapping, tetrapeptide and macromolecular substrate specificities that were partially speciesspecific (Fig. 1-4). Whereas $\alpha$-tubulin was a shared GrM substrate between species (Fig. 5), only human but not mouse NPM was cleaved by both granzymes (Fig. 6). Both hGrM and mGrM efficiently triggered apoptosis in human HeLa cells, but not in several mouse (tumor) cell lines (Fig. 7). These data show that hGrM and mGrM are structurally and functionally divergent and cannot be used interchangeably.

Using a PS-SCL and a complementary positional proteomics approach, we showed that the P1 and extended specificities of hGrM and mGrM differ substantially. The PS-SCL results showed that, whereas hGrM prefers a Leu at P1 [13, 14], mGrM favored a Met (or Nle) > Leu at this position (Fig. 1D). The results of the PS-SCL were confirmed by positional proteomics, which also revealed that Leu and Met are major P1 determinants for hGrM and mGrM (Fig. 4). Upon differential comparison between both granzymes (Fig. 4C), mGrM was better able to accommodate a Met at P1, while hGrM clearly preferred a Leu. These data are in agreement with the facts that Leu and Met are large hydrophobic residues and that the S1 pocket of hGrM which can only accommodate long, narrow hydrophobic amino acids $[34,43]$ - is conserved in $\mathrm{mGrM}$ (Fig. 1). The substrate specificity of $\mathrm{hGrM}$ and $\mathrm{mGrM}$ diverges further at the $\mathrm{P} 2$ position. PS-SCL and positional proteomics showed preferences for Ala and Pro at P2 for hGrM $[13,14]$ and $\mathrm{mGrM}$ (Fig. 1D and 4A-B). Major differences between hGrM and mGrM at the P2 position include a better tolerance to Glu, Thr, and Val by hGrM, and to Gly by mGrM (Fig. 4C). Both hGrM and mGrM displayed a clear preference for Glu at the P3 position, which was demonstrated by both PS-SCL (Fig. 1D) and positional proteomics (Fig. 4). The PS-SCL results showed that mGrM had broad preference at the P4 position (Fig. 1D), whereas in natural substrates mGrM favored a Lys or Arg at this position, the latter being discriminative for mGrM as compared to hGrM (Fig. 4). In PS-SCL, hGrM shows a clear preference for the positively charged Lys and His residues at P4 [13], which can also be deduced from positional proteomics (Fig. 4).

Aside from the non-prime subsite preferences, we show for the first time that prime site residues are also important structural determinants for GrM-substrate recognition. In our positional proteomics approach, the amino acid occupancy of the P2' position was found to be clearly discriminative between hGrM and mGrM, with hGrM accommodating Ala, Asn, Lys, Ser, Gly and Val, and mGrM accomodating Leu, Phe and Tyr to a better extent at this position (Fig. 4C). Furthermore, replacement of the putative P1'-P2' residues in mouse nucleophosmin with the corresponding residues of human nucleophosmin fully restored cleavage of mouse nucleophosmin by both hGrM and mGrM (Fig. 6). It seems unlikely that major structural differences around the GrM cleavage site between human and mouse NPM affect GrM proteolysis, since secondary structures and solvent accessibility of the GrM cleavage site regions in human and mouse NPM are predicted to be similar. The importance of prime site residues for GrM cleavage is compatible with the notion that relatively high concentrations of GrM were required for robust activity in PS-SCL (Fig. 1D).

We employed two proteomic approaches to dissect the macromolecular substrate specificities of hGrM and mGrM (Fig. 2-4 and Suppl. Table 1). Some substrates were shared, whereas others were specific for either hGrM or mGrM. Using the 2D-DIGE gel-based approach, only limited sets of hGrM (40, $1.3 \%$ of total spots) and mGrM (12, $0.5 \%$ of total spots) substrates were resolved in their species-matched cell lysates, indicating that both granzymes are highly specific. 
In the human HeLa cell lysate, $40 \mathrm{GrM}$ substrates were identified, of which 17 ( $42 \%)$ were shared by hGrM and mGrM. Using the gel-free complementary positional proteomics approach, we identified a total of 488 GrM substrates in the human K-562 cell lysate, of which 359 substrates $(\sim 74 \%)$ were shared between hGrM and mGrM. This indicates that the macromolecular substrate specificities of hGrM and $\mathrm{mGrM}$ only partially overlap. The discrepancy in the number of identified GrM substrates between the gel-free positional proteomics approach as compared with the gel-based 2D-DIGE approach is most likely due to the selection criteria and to the intrinsic higher sensitivity of gel-free proteomics [44]. Both the 2DDIGE and the COFRADIC approach showed that while hGrM and mGrM share some cleavage sites in macromolecular substrates, they also make use of unique sites, which is in agreement with the partially overlapping primary and extended substrate specificity data as determined by both the PS-SCL and the positional proteomics approach (Fig. 1D and 4, Suppl. Table 1). Interestingly, both approaches revealed only few unique mGrM substrates.

The ability of GrM to cleave specific macromolecular substrates partially depends on the substrate conservation between human and mouse, suggesting that GrM has species-specific functions (Figures 5 and 6). The multifunctional phosphoprotein NPM is essential for cell viability and plays a role in viral replication [20, 45, 46]. Recently, it has been demonstrated that cleavage of human NPM by hGrM abolishes NPM function, which has been suggested to contribute to GrM-induced cell death [20]. NPM is a species-specific substrate of GrM: murine NPM was cleaved by neither mGrM nor hGrM, whereas human NPM was cleaved by both granzymes (Fig. 6). Interestingly, human and mouse GrB can also efficiently cleave human NPM [47], but fail to cleave mouse NPM [41]. These data indicate that the cleavage and inactivation of NPM does not contribute to GrM and GrB function in mice. In contrast, the microtubule network component $\alpha$-tubulin of both human and mouse origin was cleaved by GrM of both species (Fig. 5 ). Although $\alpha$-tubulin is highly conserved between human and mouse ( $>99 \%)$, it is cleaved by hGrM and mGrM at different sites and/or with different efficiencies at specific sites (Fig. 5D and Suppl. Table 1). Apparently, targeting of $\alpha$-tubulin by GrM and subsequent disorganization of the microtubule network is conserved between human and mouse and likely contributes to cytotoxic lymphocyte-induced cell death [19].

Whereas hGrM has previously been shown to efficiently induce cell death in multiple human cell lines [17, 19, 20, 22, 34], both hGrM and mGrM did not trigger cell death in several (tumor) cell lines of mouse origin (Fig. 5). Whether or not GrM induces cell death in mice remains unclear and its lack of cytotoxicity in mouse cell lines may be due to several reasons. First, the cell death potential of GrM may depend on the type of target cell line that is used. mGrM efficiently induced cell death in human HeLa cells, indicating the apoptotic potential of this granzyme (Fig. 5). Strikingly, mGrM did not trigger apoptosis in several mouse cell lines (i.e. $\mathrm{C}_{2} \mathrm{C}_{12}, \mathrm{C} 26$, and LR7) (Fig. 5). In agreement with this, Pao and colleagues have shown that GrM is not essential for NK-cell mediated cytotoxicity against tumor targets in mice [24]. However, a more recent mouse study has demonstrated that adoptively transferred NK cells from wild-type mice, but not from GrM-deficient mice, effectively inhibit the growth of a subcutaneous tumor [18]. Second, the ability of GrM to trigger apoptosis in mouse cell lines may be hampered by the presence of specific inhibitors of GrM-mediated apoptosis such as the murine serine protease inhibitor SPI-CI, which has previously been shown to directly inhibit GrM activity [48]. Currently, no natural intracellular inhibitor of hGrM has been described. Finally, apart from $\alpha$ tubulin and NPM, it remains unknown whether murine orthologs of other known hGrM death substrates such as HSP-75, ICAD, PARP, and survivin [21-23] are cleaved by GrM in mice.

Mouse models are often employed to study the role of granzymes in vivo. GrM knockout mice have demonstrated a role for GrM in the host response against tumors [18] and cytomegalovirus infection [24]. Recently, GrM deficient mice have allowed the identification of GrM as being a key regulator of inflammation, possibly by enhancing the inflammatory cascade downstream of LPS-TLR4 signaling [10]. Whether or not these in vivo data can be directly 
extrapolated to elucidate the functions of GrM in humans remains uncertain, since the speciesspecific substrate specificities of GrM signify that caution is essential when interpreting data from mouse studies. 


\section{ACKNOWLEDGEMENTS}

We thank Dr. C.E. Hack for critical reading of this manuscript and Dr. E.L. Schneider for helpful discussions. The recombinant granzymes used in the $\mathrm{N}$ - and $\mathrm{C}$-terminal COFRADIC analyses were kindly provided by Dr. P.I. Bird. P.V.D. is a Postdoctoral Fellow of the Research Foundation - Flanders (FWO-Vlaanderen). K.P. is supported by a Ph.D. grant of the Institute for the Promotion of Innovation through Science and Technology in Flanders (IWT-Vlaanderen). This work was supported by research grants from the Fund for Scientific Research-Flanders (Belgium) [grants G.0077.06 and G.0042.07 to K.G.], the Concerted Research Actions [grant BOF07/GOA/012 to K.G.] from Ghent University and the Interuniversity Attraction Poles [grant IUAP06], the Dutch Cancer Society (KWF) [grant UU-2009-4302 to N.B.], and the Netherlands Organization for Scientific Research (NWO) [grant 916.66.044 to N.B.].

\section{CONFLICT OF INTEREST}

The authors declare no conflict of interest. 


\section{References}

1 Lieberman, J. (2010) Anatomy of a murder: how cytotoxic T cells and NK cells are activated, develop, and eliminate their targets. Immunol Rev 235, 5-9

2 Russell, J. H. and Ley, T. J. (2002) Lymphocyte-mediated cytotoxicity. Annu Rev Immunol 20, 323-370

3 Walczak, H. and Krammer, P. H. (2000) The CD95 (APO-1/Fas) and the TRAII (APO-2L) apoptosis systems. Experimental cell research 256, 58-66

4 Cullen, S. P., Brunet, M. and Martin, S. J. (2010) Granzymes in cancer and immunity. Cell death and differentiation 17, 616-623

5 Dustin, M. L. and Long, E. O. (2010) Cytotoxic immunological synapses. Immunol Rev 235, 24-34

6 Anthony, D. A., Andrews, D. M., Watt, S. V., Trapani, J. A. and Smyth, M. J. (2010) Functional dissection of the granzyme family: cell death and inflammation. Immunol Rev 235, 73-92

7 Grossman, W. J., Revell, P. A., Lu, Z. H., Johnson, H., Bredemeyer, A. J. and Ley, T. J. (2003) The orphan granzymes of humans and mice. Current opinion in immunology 15, 544-552

8 Smyth, M. J. and Trapani, J. A. (1995) Granzymes: exogenous proteinases that induce target cell apoptosis. Immunology today 16, 202-206

9 Bovenschen, N. and Kummer, J. A. (2010) Orphan granzymes find a home. Immunol Rev 235, 117-127

10 Anthony, D. A., Andrews, D. M., Chow, M., Watt, S. V., House, C., Akira, S., Bird, P. I., Trapani, J. A. and Smyth, M. J. (2010) A role for granzyme M in TLR4-driven inflammation and endotoxicosis. J Immunol 185, 1794-1803

11 Metkar, S. S., Menaa, C., Pardo, J., Wang, B., Wallich, R., Freudenberg, M., Kim, S., Raja, S. M., Shi, L., Simon, M. M. and Froelich, C. J. (2008) Human and mouse granzyme A induce a proinflammatory cytokine response. Immunity $\mathbf{2 9}$, 720-733

12 de Koning, P. J., Kummer, J. A. and Bovenschen, N. (2009) Biology of granzyme M: a serine protease with unique features. Crit Rev Immunol 29, 307-315

13 Mahrus, S. and Craik, C. S. (2005) Selective chemical functional probes of granzymes A and B reveal granzyme B is a major effector of natural killer cellmediated lysis of target cells. Chem Biol 12, 567-577

14 Mahrus, S., Kisiel, W. and Craik, C. S. (2004) Granzyme M is a regulatory protease that inactivates proteinase inhibitor 9 , an endogenous inhibitor of granzyme B. J Biol Chem 279, 54275-54282

15 de Koning, P. J., Tesselaar, K., Bovenschen, N., Colak, S., Quadir, R., Volman, T. J. and Kummer, J. A. (2010) The cytotoxic protease granzyme M is expressed by lymphocytes of both the innate and adaptive immune system. Mol Immunol 47,

16 Smyth, M. J., Sayers, T. J., Wiltrout, T., Powers, J. C. and Trapani, J. A. (1993) Met-ase: cloning and distinct chromosomal location of a serine protease preferentially expressed in human natural killer cells. J Immunol 151, 6195-6205

17 Kelly, J. M., Waterhouse, N. J., Cretney, E., Browne, K. A., Ellis, S., Trapani, J. A. and Smyth, M. J. (2004) Granzyme M mediates a novel form of perforindependent cell death. J Biol Chem 279, 22236-22242 
18 Pegram, H. J., Haynes, N. M., Smyth, M. J., Kershaw, M. H. and Darcy, P. K. (2010) Characterizing the anti-tumor function of adoptively transferred NK cells in vivo. Cancer Immunol Immunother

19 Bovenschen, N., de Koning, P. J., Quadir, R., Broekhuizen, R., Damen, J. M., Froelich, C. J., Slijper, M. and Kummer, J. A. (2008) NK cell protease granzyme $\mathrm{M}$ targets alpha-tubulin and disorganizes the microtubule network. J Immunol 180, 8184-8191

20 Cullen, S. P., Afonina, I. S., Donadini, R., Luthi, A. U., Medema, J. P., Bird, P. I. and Martin, S. J. (2009) Nucleophosmin is cleaved and inactivated by the cytotoxic granule protease granzyme $\mathrm{M}$ during natural killer cell-mediated killing. J Biol Chem 284, 5137-5147

21 Hu, D., Liu, S., Shi, L., Li, C., Wu, L. and Fan, Z. (2010) Cleavage of survivin by Granzyme M triggers degradation of the survivin-X-linked inhibitor of apoptosis protein (XIAP) complex to free caspase activity leading to cytolysis of target tumor cells. J Biol Chem 285, 18326-18335 Hua, G., Zhang, Q. and Fan, Z. (2007) Heat shock protein 75 (TRAP1) antagonizes reactive oxygen species generation and protects cells from granzyme M-mediated apoptosis. J Biol Chem 282, 20553-20560 Lu, H., Hou, Q., Zhao, T., Zhang, H., Zhang, Q., Wu, L. and Fan, Z. (2006) Granzyme M directly cleaves inhibitor of caspase-activated DNase (CAD) to unleash CAD leading to DNA fragmentation. J Immunol 177, 1171-1178 Pao, L. I., Sumaria, N., Kelly, J. M., van Dommelen, S., Cretney, E., Wallace, M. E., Anthony, D. A., Uldrich, A. P., Godfrey, D. I., Papadimitriou, J. M., Mullbacher, A., Degli-Esposti, M. A. and Smyth, M. J. (2005) Functional analysis of granzyme $\mathrm{M}$ and its role in immunity to infection. J Immunol 175, 3235-3243

25 Arnold, K., Bordoli, L., Kopp, J. and Schwede, T. (2006) The SWISS-MODEL workspace: a web-based environment for protein structure homology modelling. Bioinformatics 22, 195-201

26 Dauber, D. S., Ziermann, R., Parkin, N., Maly, D. J., Mahrus, S., Harris, J. L., Ellman, J. A., Petropoulos, C. and Craik, C. S. (2002) Altered substrate specificity of drug-resistant human immunodeficiency virus type 1 protease. Journal of virology 76, 1359-1368

27 Harris, J. L., Backes, B. J., Leonetti, F., Mahrus, S., Ellman, J. A. and Craik, C. S. (2000) Rapid and general profiling of protease specificity by using combinatorial fluorogenic substrate libraries. Proc Natl Acad Sci U S A 97, 7754-7759

28 Bovenschen, N., Quadir, R., van den Berg, A. L., Brenkman, A. B., Vandenberghe, I., Devreese, B., Joore, J. and Kummer, J. A. (2009) Granzyme K displays highly restricted substrate specificity that only partially overlaps with granzyme A. J Biol Chem 284, 3504-3512

29 Alban, A., David, S. O., Bjorkesten, L., Andersson, C., Sloge, E., Lewis, S. and Currie, I. (2003) A novel experimental design for comparative two-dimensional gel analysis: two-dimensional difference gel electrophoresis incorporating a pooled internal standard. Proteomics 3, 36-44

Staes, A., Van Damme, P., Helsens, K., Demol, H., Vandekerckhove, J. and Gevaert, K. (2008) Improved recovery of proteome-informative, protein N- 
terminal peptides by combined fractional diagonal chromatography

(COFRADIC). Proteomics 8, 1362-1370

31 Van Damme, P., Martens, L., Van Damme, J., Hugelier, K., Staes, A., Vandekerckhove, J. and Gevaert, K. (2005) Caspase-specific and nonspecific in vivo protein processing during Fas-induced apoptosis. Nat Methods 2, 771-777

32 Van Damme, P., Staes, A., Bronsoms, S., Helsens, K., Colaert, N., Timmerman, E., Aviles, F. X., Vandekerckhove, J. and Gevaert, K. Complementary positional proteomics for screening substrates of endo- and exoproteases. Nat Methods 7, 512-515

33 Ghesquiere, B., Colaert, N., Helsens, K., Dejager, L., Vanhaute, C., Verleysen, K., Kas, K., Timmerman, E., Goethals, M., Libert, C., Vandekerckhove, J. and Gevaert, K. (2009) In vitro and in vivo protein-bound tyrosine nitration characterized by diagonal chromatography. Mol Cell Proteomics 8, 2642-2652

34 Wu, L., Wang, L., Hua, G., Liu, K., Yang, X., Zhai, Y., Bartlam, M., Sun, F. and Fan, Z. (2009) Structural basis for proteolytic specificity of the human apoptosisinducing granzyme M. J Immunol 183, 421-429

35 Schechter, I. and Berger, A. (1967) On the size of the active site in proteases. I. Papain. Biochem Biophys Res Commun 27, 157-162

36 Van Damme, P., Maurer-Stroh, S., Plasman, K., Van Durme, J., Colaert, N., Timmerman, E., De Bock, P. J., Goethals, M., Rousseau, F., Schymkowitz, J., Vandekerckhove, J. and Gevaert, K. (2009) Analysis of protein processing by Nterminal proteomics reveals novel species-specific substrate determinants of granzyme B orthologs. Mol Cell Proteomics 8, 258-272

37 Ong, S. E., Blagoev, B., Kratchmarova, I., Kristensen, D. B., Steen, H., Pandey, A. and Mann, M. (2002) Stable isotope labeling by amino acids in cell culture, SILAC, as a simple and accurate approach to expression proteomics. Mol Cell Proteomics 1, 376-386

38 Colaert, N., Helsens, K., Martens, L., Vandekerckhove, J. and Gevaert, K. (2009) Improved visualization of protein consensus sequences by iceLogo. Nat Methods 6, 786-787

39 Grisendi, S., Bernardi, R., Rossi, M., Cheng, K., Khandker, L., Manova, K. and Pandolfi, P. P. (2005) Role of nucleophosmin in embryonic development and tumorigenesis. Nature 437, 147-153

40 Casciola-Rosen, L., Garcia-Calvo, M., Bull, H. G., Becker, J. W., Hines, T., Thornberry, N. A. and Rosen, A. (2007) Mouse and human granzyme B have distinct tetrapeptide specificities and abilities to recruit the bid pathway. J Biol Chem 282, 4545-4552

41 Cullen, S. P., Adrain, C., Luthi, A. U., Duriez, P. J. and Martin, S. J. (2007) Human and murine granzyme B exhibit divergent substrate preferences. J Cell Biol 176, 435-444

Kaiserman, D., Bird, C. H., Sun, J., Matthews, A., Ung, K., Whisstock, J. C., Thompson, P. E., Trapani, J. A. and Bird, P. I. (2006) The major human and mouse granzymes are structurally and functionally divergent. J Cell Biol 175, 619-630

43 Smyth, M. J., O'Connor, M. D., Trapani, J. A., Kershaw, M. H. and Brinkworth, R. I. (1996) A novel substrate-binding pocket interaction restricts the specificity 
of the human NK cell-specific serine protease, Met-ase-1. J Immunol 156, 41744181

44 Plasman, K., Van Damme, P., Kaiserman, D., Impens, F., Demeyer, K., Helsens, K., Goethals, M., Bird, P. I., Vandekerckhove, J. and Gevaert, K. (2010) Probing the efficiency of proteolytic events by positional proteomics. Mol Cell Proteomics

45 Huang, W. H., Yung, B. Y., Syu, W. J. and Lee, Y. H. (2001) The nucleolar phosphoprotein B23 interacts with hepatitis delta antigens and modulates the hepatitis delta virus RNA replication. J Biol Chem 276, 25166-25175

Okuwaki, M., Iwamatsu, A., Tsujimoto, M. and Nagata, K. (2001) Identification of nucleophosmin/B23, an acidic nucleolar protein, as a stimulatory factor for in vitro replication of adenovirus DNA complexed with viral basic core proteins. Journal of molecular biology 311, 41-55

Ulanet, D. B., Torbenson, M., Dang, C. V., Casciola-Rosen, L. and Rosen, A. (2003) Unique conformation of cancer autoantigen B23 in hepatoma: a mechanism for specificity in the autoimmune response. Proc Natl Acad Sci U S A 100, 12361-12366

48 Bots, M., Kolfschoten, I. G., Bres, S. A., Rademaker, M. T., de Roo, G. M., Kruse, M., Franken, K. L., Hahne, M., Froelich, C. J., Melief, C. J., Offringa, R. and Medema, J. P. (2005) SPI-CI and SPI-6 cooperate in the protection from effector cell-mediated cytotoxicity. Blood 105, 1153-1161 Martens, L., Hermjakob, H., Jones, P., Adamski, M., Taylor, C., States, D., Gevaert, K., Vandekerckhove, J. and Apweiler, R. (2005) PRIDE: the proteomics identifications database. Proteomics 5, 3537-3545 


\section{Footnote}

${ }^{1}$ Identified MS/MS spectra are made publicly available in the Proteomics Identifications Database (PRIDE) [49] under the accession code 15475. 


\section{Figure and table legends}

FIG. 1. The primary and extended specificities of human and mouse GrM are distinct.

(A) Alignment of hGrM and $\mathrm{mGrM}$ primary sequence. Residues boxed in grey are shared amino acids between hGrM and mGrM, those boxed in black are residues that form the catalytic triad. The S1-S2-S3-S4 amino acids are highlighted in yellow, green, red and blue, respectively. (B) Crystal structure of hGrM [34]. Based on the structure of hGrM, a homology model for mGrM was generated (Swiss-Prot). S1-S4 amino acids are indicated. (C) Michaelis plots of peptide substrate KVPL-pNA hydrolysis by different concentrations of human (h) and mouse (m) GrM. Calculated $\mathrm{k}_{\mathrm{cat}} / \mathrm{K}_{\mathrm{m}}$ values are indicated. Results are representative of two independent triplicate experiments. (D) The primary specificity of mGrM was determined using a P1-ACC diverse tetrapeptide library. The extended specificity of mGrM was determined using a P1 fixed MetACC tetrapeptide library. The $y$ axis represents the rate of ACC production as a percentage of the maximum rate observed in each experiment. The $x$ axis shows the positioned $\mathrm{P} 1$ amino acids as represented by the one-letter code. Data is representative of three separate experiments.

FIG. 2. Human and mouse GrM display highly restricted macromolecular substrate specificities that only partially overlap. (A) HeLa cell freeze/thaw lysates $(100 \mu \mathrm{g})$ were incubated with $1 \mu \mathrm{M}$ hGrM (green) or $1 \mu \mathrm{M}$ human GrM-SA (hGrM-SA) (red) for $1 \mathrm{~h}$ at $37^{\circ} \mathrm{C}$. The samples were then subjected to 2D-DIGE. Protein spots shared between the two samples appear yellow. Protein spots with decreased abundance after protease digestion (GrM substrates) are red, while new spots (cleavage products) that appear after protease digestion are green. (B) $\mathrm{C}_{2} \mathrm{C}_{12}$ cell freeze/thaw lysates $(100 \mu \mathrm{g})$ were incubated with $1 \mu \mathrm{M} \mathrm{mGrM}$ (green) or $1 \mu \mathrm{M}$ mouse GrM-SA (mGrM-SA) (red) for $1 \mathrm{~h}$ at $37^{\circ} \mathrm{C}$ and subjected to 2D-DIGE. These results are representative for five independent experiments. (C) Human GrM/GrM-SA and mouse GrM/GrM-SA gels from HeLa lysate were matched, and human GrM-SA/GrM log peak volume ratios ( $y$ axis) were plotted against mouse GrM-SA/GrM log peak volume ratios ( $x$ axis). Protein spots are labeled red (intact substrates), green (cleavage fragments), or yellow (not affected proteome). Protein spot intensity was considered to be changed when peak volume ratios were 2fold (dotted lines) and $\mathrm{p}<0.05$. (D) Human GrM/GrM-SA and mouse GrM/GrM-SA gels from $\mathrm{C}_{2} \mathrm{C}_{12}$ lysate were matched, and human GrM-SA/GrM log peak volume ratios ( $y$ axis) were plotted against mouse GrM-SA/GrM log peak volume ratios ( $x$ axis).

FIG. 3. Illustration of the different categories of isolated terminal peptides found in $\mathbf{K}-562$ lysates. SILAC-labeled K-562 freeze-thaw lysates were treated with $\mathrm{hGrM}\left({ }^{12} \mathrm{C}_{6}\right)$, mGrM $\left({ }^{13} \mathrm{C}_{6}\right)$ or left untreated $\left({ }^{13} \mathrm{C}_{6}{ }^{15} \mathrm{~N}_{4}\right)$. (A) Unaltered database annotated protein-N-termini: these peptides were identified as $\mathrm{Ac}^{-}{ }^{2}$ TSKGPEEEHPSVTLFR $^{17}$ (Ac denotes $\alpha$-acetylated amino group) from aminoacylase-1 and the ion intensities of their three different forms are about equal, indicating that this peptide was not affected by GrM. (B) Unique neo-N-terminus (QEAL $\downarrow$ AcD3${ }^{349} \mathrm{AKLKEEEER}^{357}$, AcD3 denotes a trideutero-acetylated $\alpha$-amino group), generated only by hGrM in the eukaryotic translation initiation factor 5B. (C) Unique neo-N-terminus (RNGL $\downarrow$ AcD3 $-{ }^{61}$ LAALKKALAAGGYDVEKNNSR ${ }^{82}$ ) generated exclusively through the action of mGrM in histone H1.5. (D) Neo-N-termini of the serine/arginine repetitive matrix protein 2 (LAAL $\downarrow A c D 3-{ }^{2310}$ SLTGSGTPPTAANYPSSSR ${ }^{2328}$ ) generated with similar efficiency by hGrM and $\mathrm{mGrM}$. (E) Unaffected protein C-termini were identified as differentially butyrylated${ }^{72}$ LAKADGIVSKNF $^{83}$ peptides of which the ion intensities are about equal $(\mathrm{L} / \mathrm{M}, \mathrm{L} / \mathrm{H}$, and $\mathrm{M} / \mathrm{H}$ ratios of $1.1,1$, and 1.1 respectively). (F) $\mathrm{A}$ neo-C terminus $\mathrm{But}^{12 / 13} \mathrm{C}_{4^{-}}$ ${ }^{416} \mathrm{KNLDVMKEAM}{ }^{425} \downarrow$ VQAE $\left(\mathrm{But}^{12 / 13} \mathrm{C}_{4}\right.$ denotes an $\alpha$-amino group modified by 4 carbon- 12 or 4 carbon-13 butyric acid) originating from the Nucleolar protein 56 was identified, according to the ion intensities, approx. 2.5 times more efficiently cleaved by hGrM than by mGrM. In a separate peptide fraction, the neo-N-terminus generated after cleavage at $\mathrm{KEAM}^{425} \downarrow$ was also 
identified following GrM treatment with a similar $\mathrm{hGrM} / \mathrm{mGrM}$ ratio of 2.4 (See also Suppl.Table 1).

FIG. 4. IceLogo representations of the cleavage sites identified by proteome-wide screening for GrM substrates. IceLogo representations were created using the respectively 220 (A) and 72 (B) Leu/Met P1-containing cleavage motifs of identified neo-termini exhibiting hGrM/mGrM cleavage ratios deviating at least $50 \%$ of the mean (i.e. preferred/unique $\mathrm{hGrM}$ (A) or preferred/unique mGrM cleavages (B)). Multiple sequence alignments [38] of peptide substrate motifs are given as 1, 2, 3 to 8 corresponding to cleavage site residues P4, P3, P2 to P4' with the P1 cleavage site indicated as position 4 . Statistically significant residues with a p-value threshold of 0.05 are plotted. The amino acids heights are indicative for their degree of conservation at the indicated position. The frequency of the amino acid occurrence at each position in the sequence set was compared with the human Swissprot 56.0 database. Residues with a statistically significant lower frequency of occurrence as compared to SwissProt are indicated at the negative site of the $x$ axis. Residues with a $0 \%$ occurrence are colored in the lightest greyscale. (C) A differential iceLogo representation was created using the P1 Leu (248) and P1 Met (44) containing cleavage motifs of identified neo-termini with corresponding hGrM and/or mGrM cleavage ratios deviating at least $50 \%$ of the mean. Statistically significant residues with a p-value threshold of 0.01 around the P1 Leu or P1 Met are plotted with the size of the amino acids proportional to the difference observed in cleavage efficiency between hGrM and $\mathrm{mGrM}$ on their respective substrates. On the $y$ axis the height of the symbol is proportional to the difference in occurrence of amino acid usage in more efficient hGrM yersus more efficient mGrM cleavage site motifs.

FIG. 5. Human and mouse GrM cleave $\alpha$-tubulin with similar efficiency. (A) Cell lysates derived from HeLa (human) or $\mathrm{C}_{2} \mathrm{C}_{12}$ (mouse) cells were treated with hGrM or mGrM (400 nM) for indicated lengths of time or with different concentrations of hGrM or mGrM (0-400 nM). Catalytically inactive GrM-SA served as a negative control. Reactions were separated by SDSPAGE and analyzed by immunoblotting, using an antibody against $\alpha$-tubulin or $\beta$-tubulin (which served as a loading control, since no significant cleavage could be observed at the conditions assayed). (B) Signal intensities of protein bands were quantified by densitometry, $\alpha$-tubulin/ $\beta$ tubulin ratios were calculated, and plotted against time or GrM concentration. (C) Both HeLa and $\mathrm{C}_{2} \mathrm{C}_{12}$ lysates were incubated for $4 \mathrm{~h}$ at $37^{\circ} \mathrm{C}$ with either human (h) or mouse (m) GrM (400 nM). Samples were immunoblotted using an antibody against $\alpha$-tubulin or $\beta$-tubulin (loading control). Results are representative of three independent experiments. (D) Purified recombinant human and mouse His- $\alpha$-tubulin were incubated for $2 \mathrm{~h}$ at $37^{\circ} \mathrm{C}$ with indicated concentrations of hGrM or $\mathrm{mGrM}$. Reactions were separated on SDS-PAGE and visualized by Simply Blue protein staining. His- $\alpha$-tubulin and GrM are indicated, arrows indicate cleavage products. Results are representative of three independent experiments. (E) Alignment of human and mouse $\alpha$-tubulin. Residues boxed in grey are the shared amino acids between human and mouse $\alpha$-tubulin. Boxed in black is one known hGrM cleavage site [19].

FIG. 6. Human and mouse GrM cleave human but not mouse nucleophosmin. (A) Cell lysates derived from HeLa (human) or $\mathrm{C}_{2} \mathrm{C}_{12}$ (mouse) cells were treated with hGrM or mGrM $(400 \mathrm{nM})$ for indicated lengths of time or with different concentrations of hGrM or mGrM (0-400 $\mathrm{nM}$ ). Reactions were separated by SDS-PAGE and analyzed by immunoblotting, using an antibody against NPM or $\beta$-tubulin (which served as a loading control, since no significant cleavage could be observed at the conditions assayed). (B) Signal intensities of protein bands were quantified by densitometry, NPM/ $\beta$-tubulin ratios were calculated, and plotted against time or GrM concentration. (C) Both HeLa and $\mathrm{C}_{2} \mathrm{C}_{12}$ lysates were incubated for $4 \mathrm{~h}$ at $37^{\circ} \mathrm{C}$ with either human (h) or mouse (m) GrM (400 nM). Samples were immunoblotted using an antibody 
against NPM or $\beta$-tubulin (loading control). Results are representative of three independent experiments. (D) Recombinant human and mouse NPM were incubated for $2 \mathrm{~h}$ at $37^{\circ} \mathrm{C}$ with the indicated concentrations of hGrM or mGrM. Reactions were separated on SDS-PAGE and visualized by Simply Blue staining. Results are representative of three independent experiments.

(E) Alignment of human and mouse NPM. Residues boxed in grey are the shared amino acids between human and mouse NPM. Boxed in dark grey is the known hGrM cleavage site [20]. (F) Alignment of the GrM cleavage site in human and mouse NPM. The P1-P4 are completely conserved between mouse and human, but the P1' and P2' residues differ. Different mutants of mouse NPM generated are depicted. (G) Purified recombinant wild type (wt) human NPM (hNPM), wt mouse NPM (mNPM) and different NPM mutants were incubated for $2 \mathrm{~h}$ at $37^{\circ} \mathrm{C}$ with the indicated concentrations of human (h) or mouse (m) GrM. Products were separated by SDS-PAGE and visualized by Simply Blue staining. Results are representative of four independent experiments.

FIG. 7. Human and mouse GrM trigger cell death in human HeLa but not mouse C2C12 tumor cells. Human HeLa cells (A) or mouse $\mathrm{C}_{2} \mathrm{C}_{12}$ cells (B) were treated with sublytic concentrations of SLO and various concentrations of hGrM, mGrM or their catalytically inactive mutants (GrM-SA), for 30 min. Cells were treated with hGrB as a positive control. After washing, cells were incubated for another $20 \mathrm{~h}$ at $37^{\circ} \mathrm{C}$. Cell viability was determined using flow cytometry, with AnnexinV and PI negative cells considered viable. 
A

$\begin{array}{lll}\text { Mouse IIGGREAVPHSRPYMASLQKAKSHVCGGVLVHRKWVLTAA } & 40 \\ \text { Human IIGGREVIPHSRPYMASLQRNGSHLCGGVLVHPKWVLTAA } & 40\end{array}$

Mouse HCLSEPLQNL̄KLLLGLHNLLHDLQDPGLTFYIREATIKḦ̈GY 80

Human 1 CLAQRMAQLRLVLGLHTL---DSPGLTFHIKAAIQHPRY 77

Mouse NH--KYENDLALIKLDRRV̄QPSKNVKPLALPRK̈PRSKRPĀE 118 Human KPVPALENDLALLQLDGKVKPSRTIRPLALPSK-RQVVAA 116

Mouse GTWCSTAGWGMTHQGGPRARALLEELDLRVLDTQMCNNSRE 158 15 Mouse WNGVIIDSMLCLKÄGSKSQAPCKGDSGGPLVCGKGQV̄-DG 197 Mouse ILSESSKTCTDIFKPPVATAVAPYSSWIRKVIGRWSPQSIV 238 Human VL FSSR

C

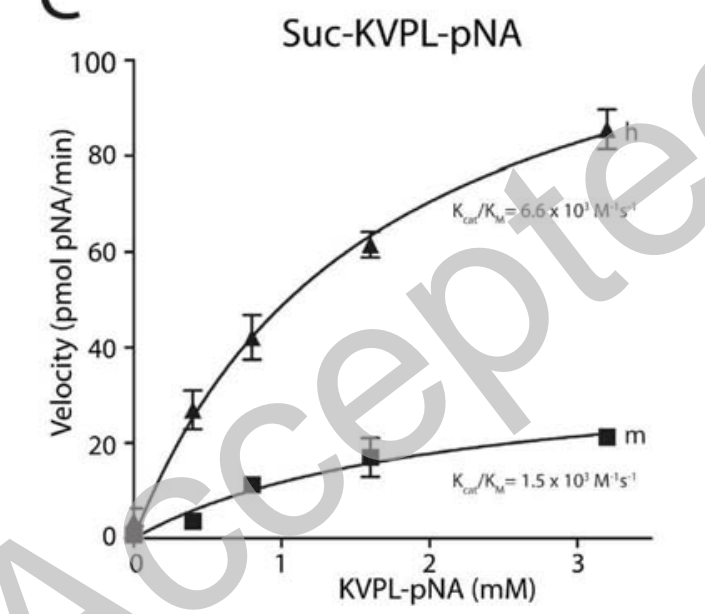

B

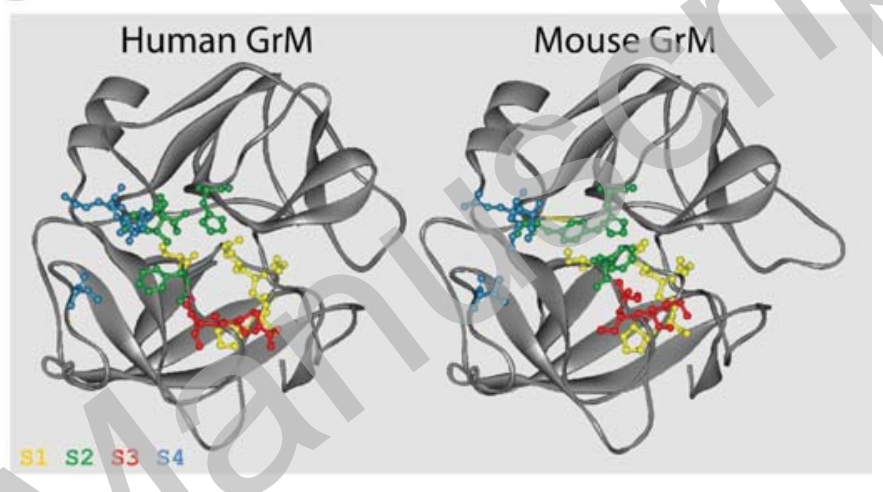

D
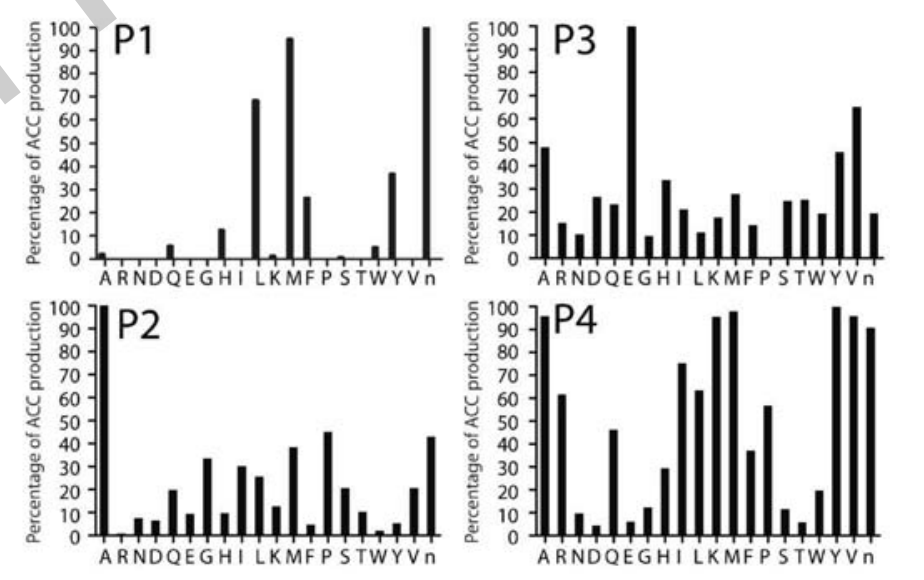
A

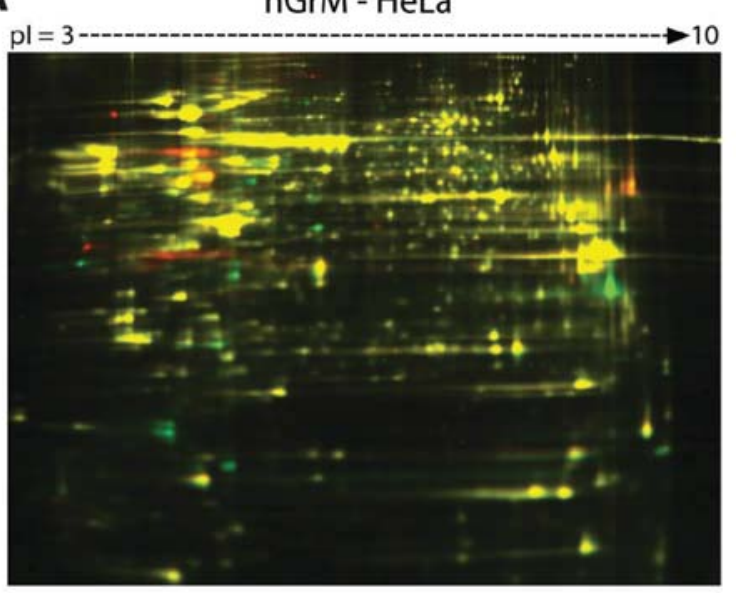

C

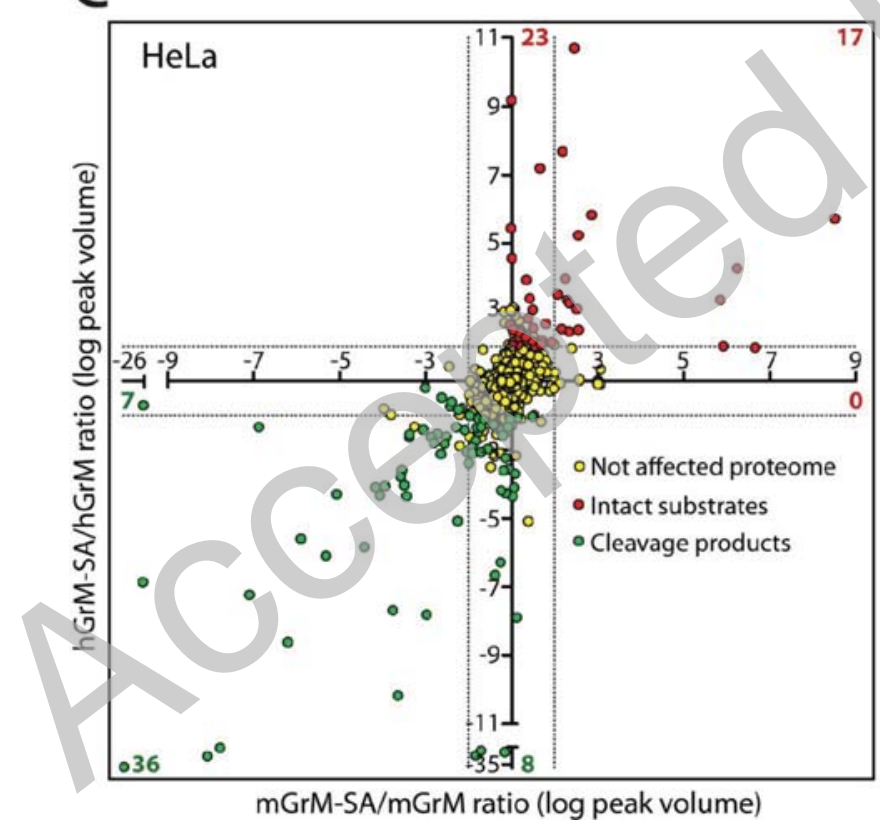

B

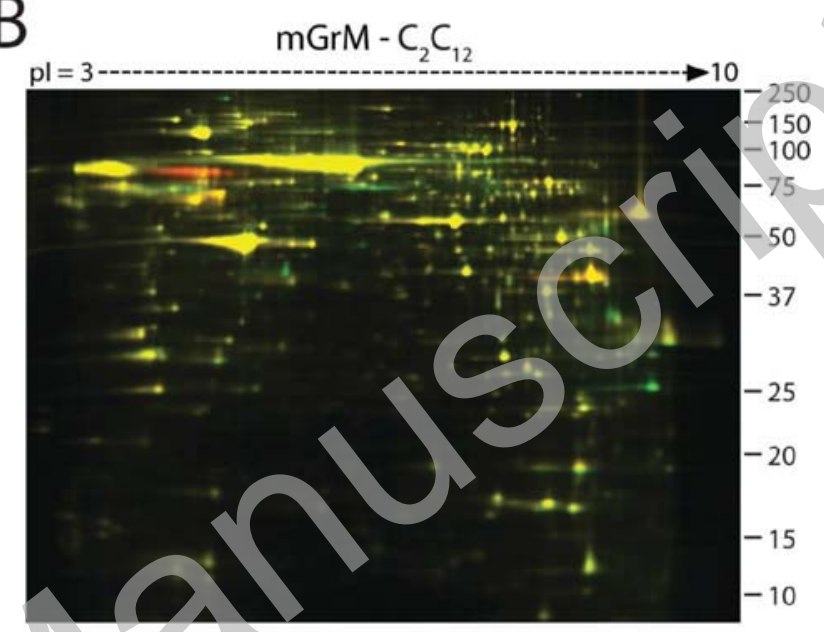

D

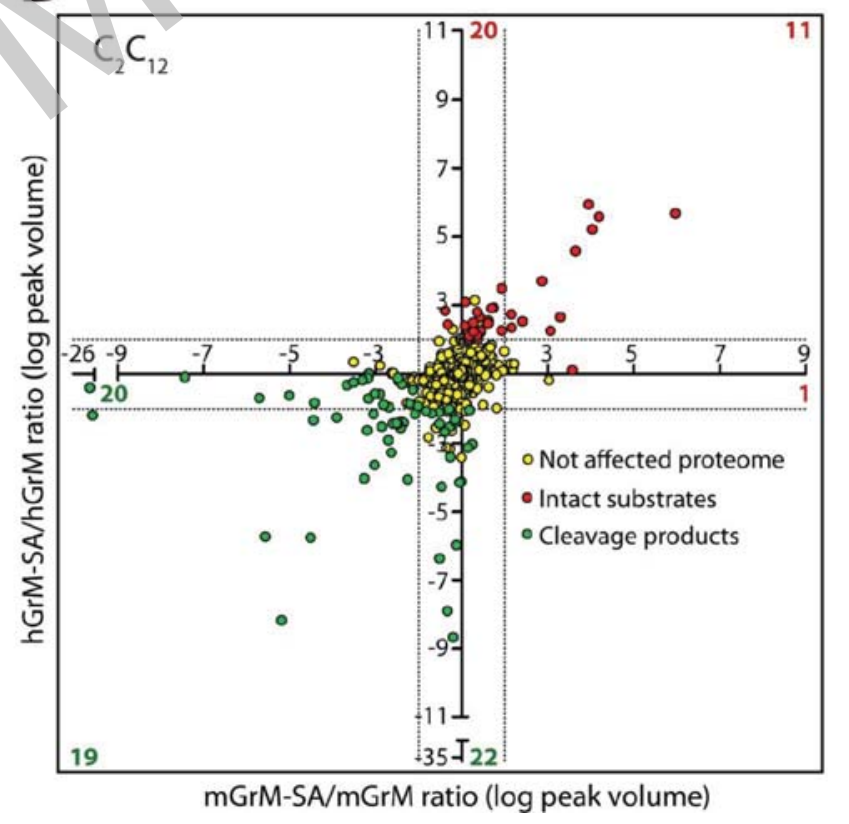

Licenced copy. Copying is not permitted, except with prior permission and as allowed by law. (C) 2011 The Authors Journal compilation (C) 2011 Portland Press Limited 
Figure 3

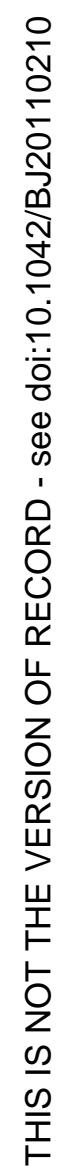
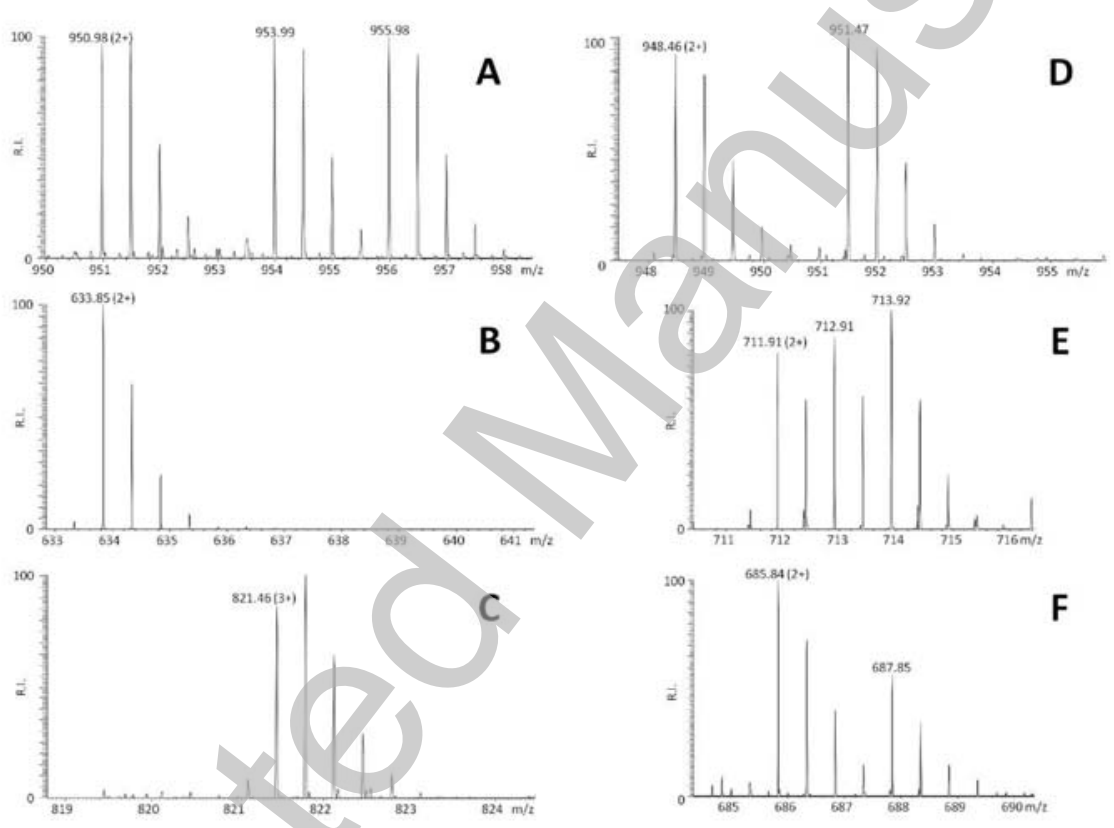

Licenced copy. Copying is not permitted, except with prior permission and as allowed by law. 

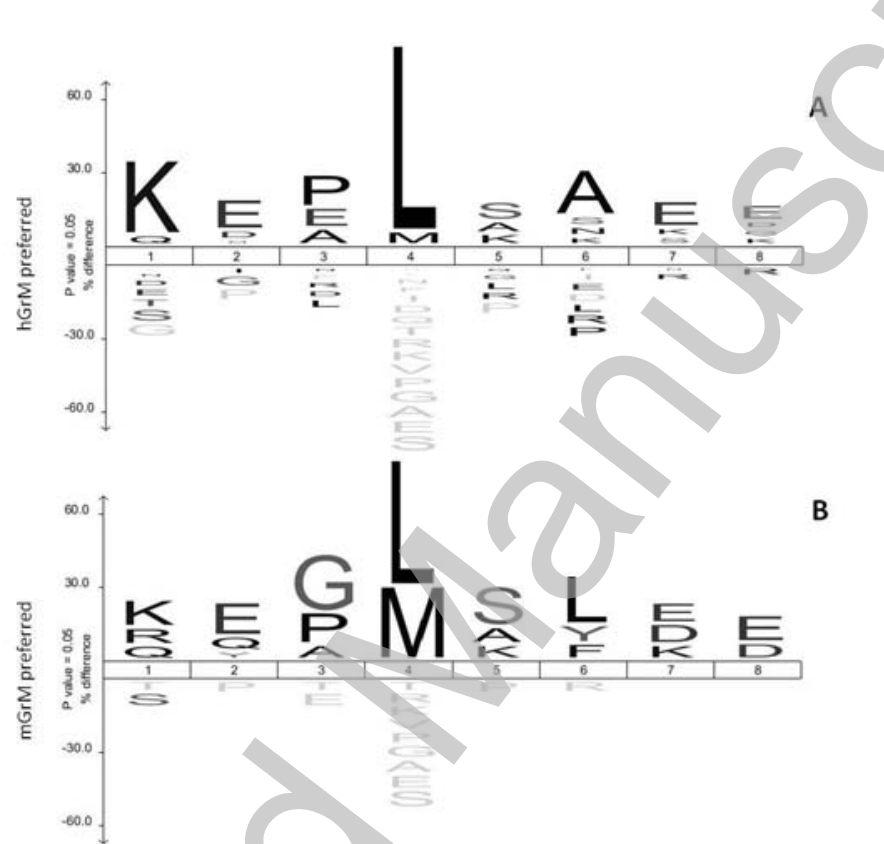

B

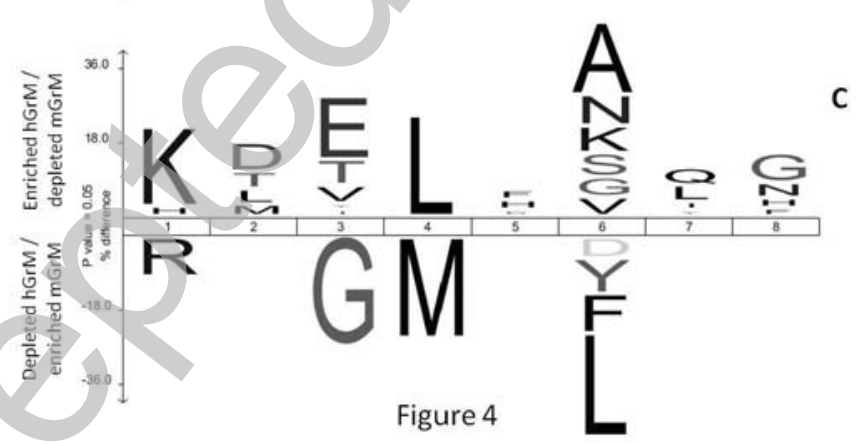


A

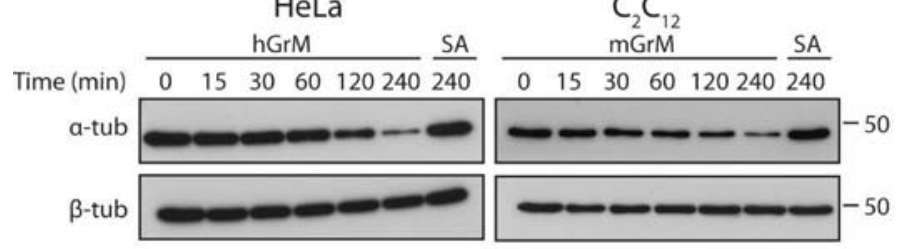

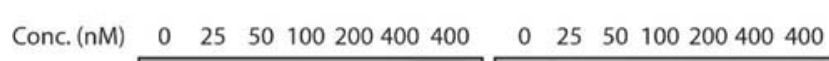

a-tub

$\beta$-tub

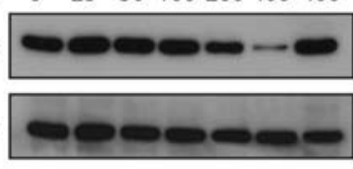

D

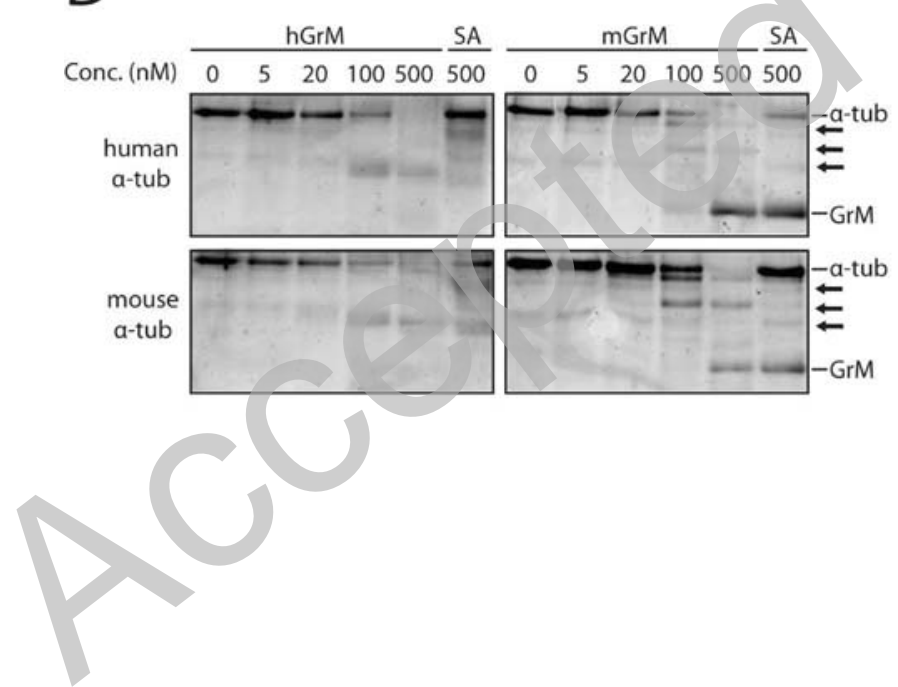

E

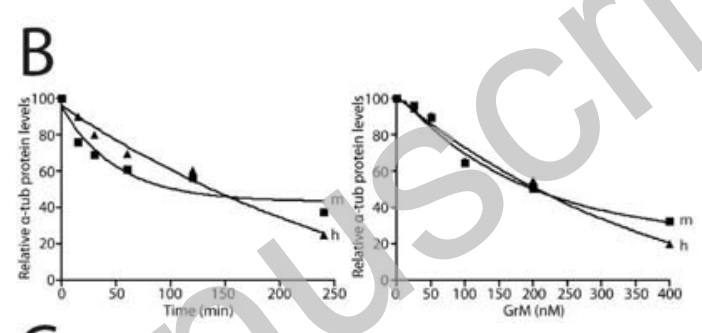

C

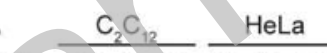

GrM SA h $\mathrm{m}$ SA h m

a-tub $\square--50$

$\beta$-tub

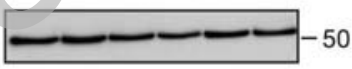

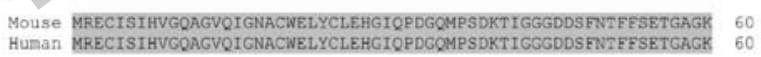

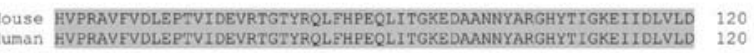
$\begin{array}{lll}\text { Youse RIRKLADQCTGLQGFLVEHSFGGGTGSGFTSLLMERLSVDYGKKSKLEFSTYPAPQVSTA } & 180 \\ \text { Human RIRKLADOCTGLOGFLVFHSFGGGTGSGFTSLLMERLSVDYGKKSKLEFSIYPAPQVSTA } & 180\end{array}$

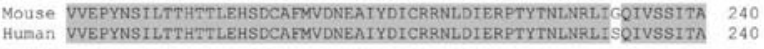

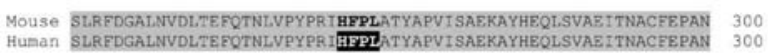

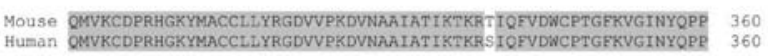

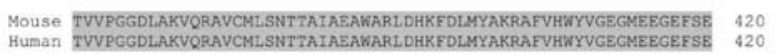
Mouse AREDMAALEKDYEEVGVDSVEGEGEEEGEEY
HuMan AREDMAALEKDYEEVGVDSVEGEGEEEGEEY 
A

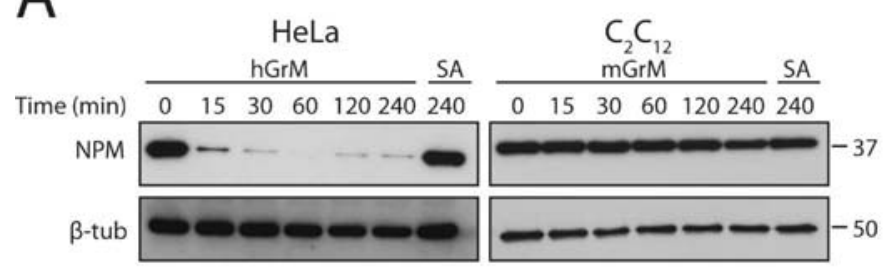

Conc. (nM) $\quad 0 \quad 25 \quad 50100200400400 \quad 0 \quad 25 \quad 50100200400400$

NPM

$\beta$-tub

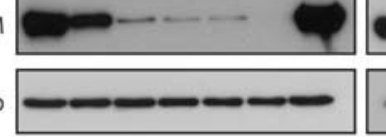

D

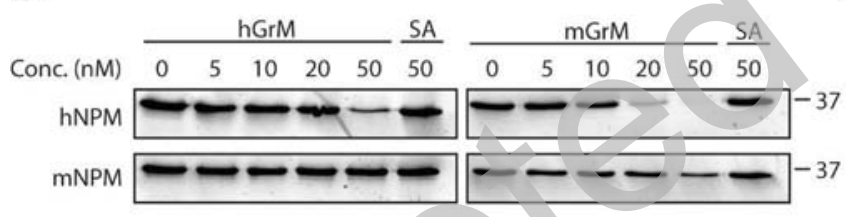

$\mathrm{F}$

MNPM 152 PQKKVKLDED 161 hNPM 152 PQKKVKLAAD 161 mNPM P1' 152 POKKVKLAED 161

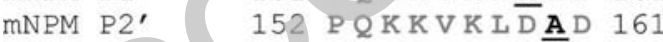

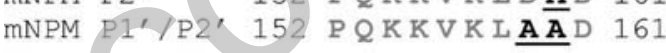

$G$
B

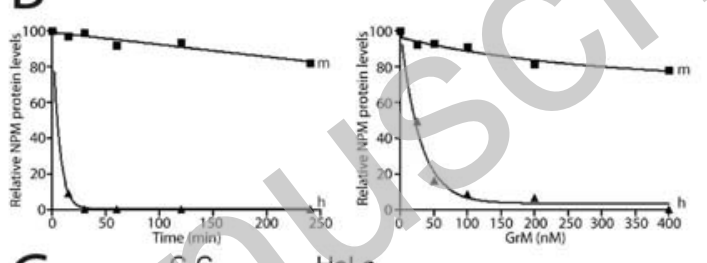

C $\frac{\mathrm{C}_{2} \mathrm{C}_{12}}{\mathrm{SA} m} \frac{\text { HeLa }}{\mathrm{SAh} \mathrm{m}}$

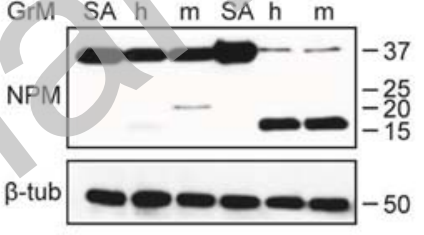

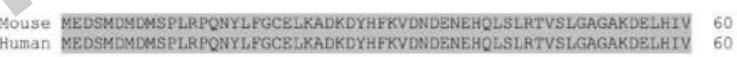
Mouse EAEAMNYEGSPIKVTLANLKMSVQPTVSIGGEEITPPVVLRLKCGSGPVHISGOHLVAVE
120

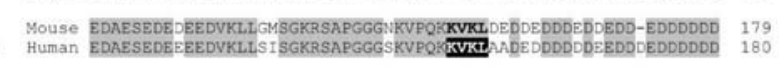

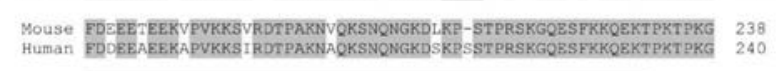

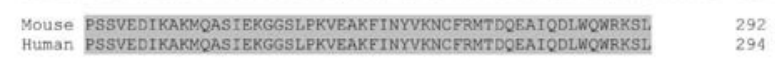
MNPM INPMP1' $m N P M P 2^{\prime}$ MNPMP1'/P2' hNPM

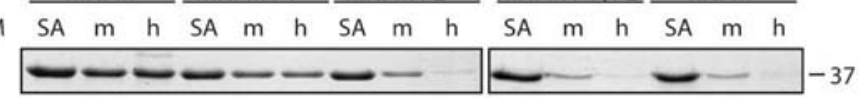


A

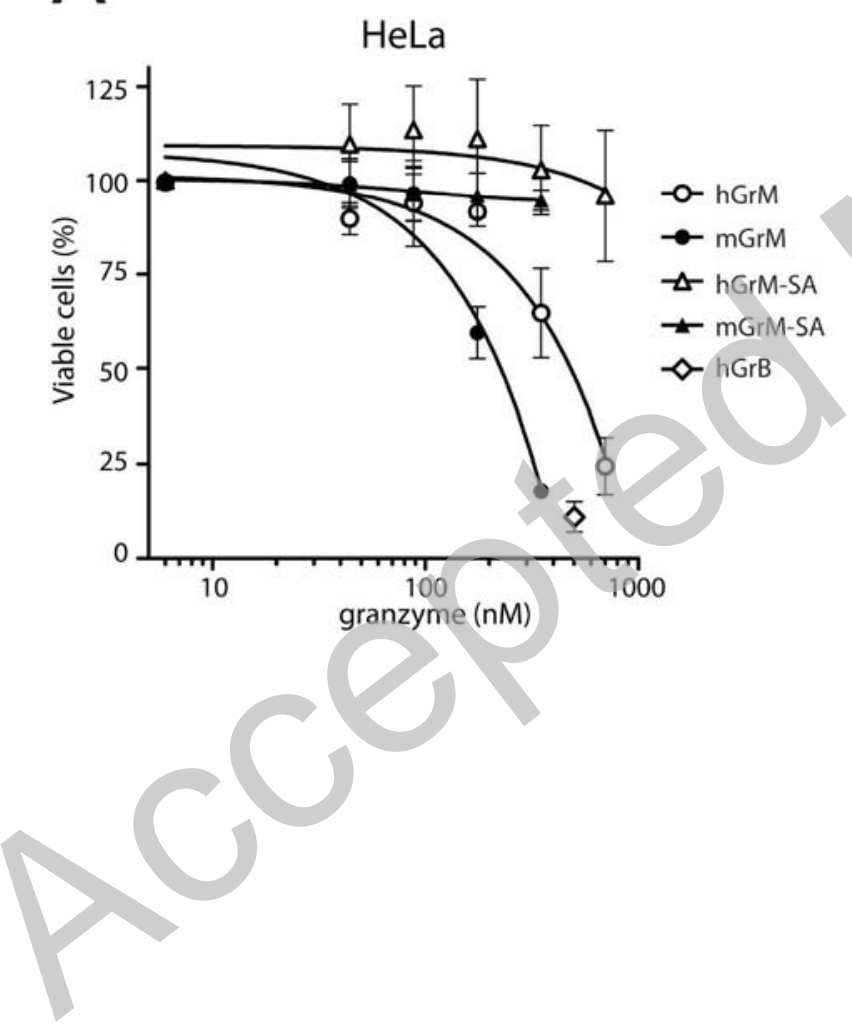

B

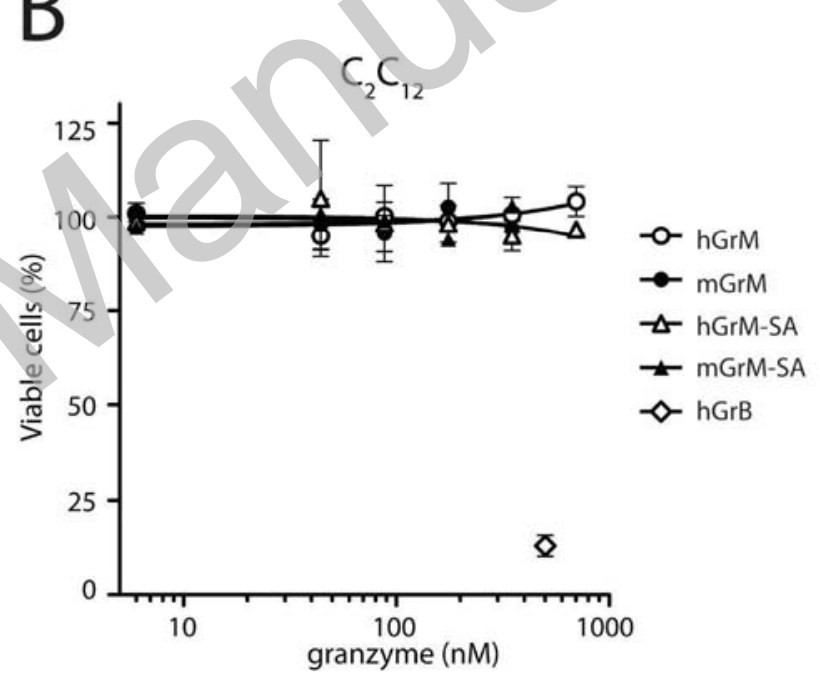

